Pacific

Journal of

Mathematics

\title{
HOMOMORPHISMS ON INFINITE DIRECT PRODUCTS OF GROUPS, RINGS AND MONOIDS
}

GEORGE M. BERGMAN 


\title{
HOMOMORPHISMS ON INFINITE DIRECT PRODUCTS OF GROUPS, RINGS AND MONOIDS
}

\author{
GEORGE M. BERGMAN
}

\begin{abstract}
We study properties of a group, abelian group, ring, or monoid $B$ which (a) guarantee that every homomorphism from an infinite direct product $\prod_{I} A_{i}$ of objects of the same sort onto $B$ factors through the direct product of finitely many ultraproducts of the $A_{i}$ (possibly after composition with the natural map $B \rightarrow B / Z(B)$ or some variant), and/or (b) guarantee that when a map does so factor (and the index set has reasonable cardinality), the ultrafilters involved must be principal.

A number of open questions and topics for further investigation are noted.
\end{abstract}

\section{Introduction}

A direct product $\prod_{i \in I} A_{i}$ of infinitely many nontrivial algebraic structures is in general a "big" object: it has at least continuum cardinality, and if the operations of the $A_{i}$ include a vector-space structure, it has at least continuum dimension. But there are many situations where the set of homomorphisms from such a product to a fixed object $B$ is unexpectedly restricted.

The poster child for this phenomenon is the case where the objects are abelian groups, and $B$ is the infinite cyclic group. In that situation, if the index set $I$ is countable (or, indeed, of less than an enormous cardinality - some details are recalled in Section 4), then every homomorphism $\prod_{i \in I} A_{i} \rightarrow B$ factors through the projection of $\prod_{i \in I} A_{i}$ onto the product of finitely many of the $A_{i}$. An abelian group $B$ which, like the infinite cyclic group, has this property, is called "slender". Slender groups have been completely characterized [Nunke 1961], and slender modules over general rings have been studied.

Archived at http://arxiv.org/abs/1406.1932. After publication, any updates, errata, related references, etc., found will be recorded at http://math.berkeley.edu/ gbergman/papers/.

MSC2010: primary 03C20, 08B25, 20A15, 20K25, 20M15, 17A01; secondary 20K40, 22B05, 16B70, $16 \mathrm{P} 60$.

Keywords: homomorphism on an infinite direct product, ultraproduct, slender group, algebraically compact group, cotorsion abelian group. 
Recent work [Bergman and Nahlus 2011 and 2012; Bergman 2014] on factorization properties of homomorphisms on infinite direct products of not-necessarilyassociative algebras (motivated by the case of Lie algebras) has turned up interesting variants on the above sort of behavior.

First, it turns out that in that context, a useful way to prove every surjective homomorphism $\prod_{i \in I} A_{i} \rightarrow B$ factors through finitely many of the $A_{i}$ is by proving (a) that every such homomorphism factors through the product of finitely many ultraproducts of the $A_{i}$, and also (b) that whenever one has a map that factors in that way, the ultrafilters involved must be principal. In this note, we shall consider each of conditions (a) and (b) on an object $B$ as of separate interest.

Secondly, we found that in many cases, though one cannot say that every surjective homomorphism from a direct product to $B$ will itself factor in one of these ways, one can say that for every such homomorphism $\prod_{i \in I} A_{i} \rightarrow B$, the induced homomorphism $\prod_{i \in I} A_{i} \rightarrow B / Z(B)$ so factors, where $Z(B)$ denotes the zero-multiplication ideal, $\{b \in B \mid b B=B b=\{0\}\}$ (which for $B$ a Lie algebra is the center of $B$ ). In the next section, we shall get similar results for groups, with $Z(B)$ the center of the group $B$. (Note that these statements do not say that every surjective homomorphism $\prod_{i \in I} A_{i} \rightarrow B / Z(B)$ factors as stated; such a factorization is asserted only when the homomorphism $\prod_{i \in I} A_{i} \rightarrow B / Z(B)$ can be lifted to a homomorphism $\prod_{i \in I} A_{i} \rightarrow B$.) Maalouf [2014] abstracts this property, and strengthens some of the results of the papers cited.

In the classical case of abelian groups (and its generalization to modules), the condition on an object $B$ that every homomorphism from an infinite product onto $B$ yield a factorization through finitely many of the $A_{i}$, and the corresponding condition for homomorphisms into $B$, are equivalent. Indeed, from any homomorphism $\prod_{i \in I} A_{i} \rightarrow B$, one can get, in an obvious way, a surjective homomorphism $B \times \prod_{i \in I} A_{i} \rightarrow B$, and the original homomorphism factors through finitely many of the $A_{i}$ if and only if that surjective map factors through $B$ and finitely many $A_{i}$. This observation uses implicitly the fact that one can add homomorphisms of abelian groups - in this case, the map $B \times \prod_{i \in I} A_{i} \rightarrow B$ induced by the given map on the one hand, and the projection to $B$ on the other. But one cannot do this for homomorphisms of noncommutative groups, of algebras, etc.; so for these, the condition involving arbitrary maps and the condition involving surjective maps are not equivalent. In these cases, the condition on $B$ defined in terms of surjective homomorphisms is the more informative. Once one has characterized those $B$ for which all surjective homomorphisms $\prod_{i \in I} A_{i} \rightarrow B$ yield such a factorization, one can, if one wishes, characterize the $B$ with the corresponding property for general 
homomorphisms as the objects all of whose subobjects have the the property for surjections.

In stating results of the sort we shall obtain, one has a choice between (i) saying that if a structure $B$ does not have one or another of a list of "messy" properties, then every homomorphism from an infinite direct product onto $B$ leads to a certain kind of factorization, or (ii) the contrapositive statement, that if there exists a homomorphism onto $B$ that does not so factor, then $B$ has one of those messy properties. Each approach has its plusses and minuses; here I have followed (ii), because it seems more straightforward to understand how a non-factorable map forces $B$ to have a messy property than to show that the absence of certain messy properties implies that all maps factor; and also because some of the conditions on $B$ come in several versions, and I find it easier to parse a statement having a single hypothesis and several conclusions than one with several alternative hypotheses giving a single conclusion. (But the above choice also has its awkward aspects; I can't say which is really best.)

In Sections 2-4, we shall study the case where our structures are not-necessarilyabelian groups, in Sections 5-7, abelian groups, then, briefly, in Section 8 and Section 9, rings and monoids. In Section 10 we note why lattices are likely to be another case worth examining.

For a short review, for the nonspecialist, of the concepts of filter, ultrafilter and ultraproduct, see [Bergman and Nahlus 2011, Appendix A]; and for measurable cardinals $\kappa$, and $\kappa$-complete ultrafilters, which come up in Sections $4-5$ below, [ibid., Appendix B]. For detailed developments of these concepts see, e.g., [Chang and Keisler 1990] or [Comfort and Negrepontis 1974].

We remark that there is in the literature a concept of "noncommutative slender group" that is quite different from the subject of Sections 2-4 below. The concept so named can be arrived at by regarding the infinite direct product in the definition of a slender abelian group as a completed direct sum, and using in the noncommutative case, instead of the direct product, an analogously completed noncommutative coproduct. For work on that topic see [Shelah and Strüngmann 2001] and references given there.

\section{Factoring group homomorphisms through finitely many ultraproducts.}

Let $\left(G_{i}\right)_{i \in I}$ be a family of groups. By the support of an element $g=\left(g_{i}\right)_{i \in I} \in$ $\prod_{i \in I} G_{i}$, we will understand the set

$$
\operatorname{supp}(g)=\left\{i \in I \mid g_{i} \neq e\right\} \subseteq I .
$$

Given any subset $S \subseteq I$, we shall identify $\prod_{i \in S} G_{i}$ in the obvious way with the subgroup of $\prod_{i \in I} G_{i}$ consisting of elements whose support is contained in $S$. In 
particular, for $g \in \prod_{i \in I} G_{i}$, the statement $g \in \prod_{i \in S} G_{i}$ will mean $\operatorname{supp}(g) \subseteq S$, and the statement $g \in G_{i}$ will mean $\operatorname{supp}(g) \subseteq\{i\}$.

Whereas the theory of slender abelian groups is based on delicate structural properties of those groups, most of our results on nonabelian groups will be based on a much simpler observation: Elements of $\prod_{i \in I} G_{i}$ with disjoint supports centralize one another. As a quick example, it is not hard to see that if $B$ is a simple nonabelian group, and we have any surjective homomorphism $f: \prod_{i \in I} G_{i} \rightarrow B$, then for each $S \subseteq I$, the map $f$ must annihilate one of the mutually centralizing subgroups $\prod_{i \in S} G_{i}$ and $\prod_{i \in S-I} G_{i}$. From this one can deduce that the subsets $S \subseteq I$ such that $f$ factors through the projection $\prod_{i \in I} G_{i} \rightarrow \prod_{i \in S} G_{i}$ form an ultrafilter (principal or nonprincipal) on $I$.

In the opposite direction, however, if we take for $B$ a cyclic group of prime order $p$ (thus losing the leverage provided by noncommutativity), and let all the $G_{i}$ be copies of that group, then by linear algebra over the field of $p$ elements, there exist homomorphisms $\prod_{i \in I} G_{i} \rightarrow B$ that send every $G_{i}$ onto $B$, and hence don't factor through any proper subproduct $\prod_{i \in S} G_{i}$.

As indicated in the introduction, we shall get around the problem created (as above) by commutativity by composing homomorphisms $\prod_{i \in I} G_{i} \rightarrow B$ with the quotient map $B \rightarrow B / Z(B)$, where $Z(B)$ is the center of $B$. Given a homomorphism $f: \prod_{i \in I} G_{i} \rightarrow B$, the key to our considerations will be the family of subsets

$$
\begin{aligned}
\mathscr{F}= & \left\{S \subseteq I \mid \text { the composite map } \prod_{i \in I} G_{i} \rightarrow B \rightarrow B / Z(B)\right. \\
& \left.\quad \text { factors through the projection } \prod_{i \in I} G_{i} \rightarrow \prod_{i \in S} G_{i}\right\} \\
= & \left\{S \subseteq I \mid f\left(\prod_{i \in I-S} G_{i}\right) \subseteq Z(B)\right\} .
\end{aligned}
$$

It is easy to see that $\mathscr{F}$, so defined, is a filter on $I$, and that if we write

$$
\pi: B \rightarrow B / Z(B)
$$

for the quotient map, then $\mathscr{F}$ is the largest filter such that $\pi f: \prod_{i \in I} G_{i} \rightarrow B / Z(B)$ factors through the reduced product $\prod_{i \in I} G_{i} / \mathscr{F}$. (The above observation, and the next few, do not yet use the fact that we are working with a map of the form $\pi f$, but only that we are considering a homomorphism on a product group. The fact that our map has the form $\pi f$ will become significant starting with Lemma 1 below.)

If the filter $\mathscr{F}$ of (2) is a finite intersection of distinct ultrafilters, $u_{0} \cap \cdots \cap u_{n-1}$, then $\prod_{i \in I} G_{i} / \mathscr{F} \cong \prod_{i \in I} G_{i} / \mathcal{U}_{0} \times \cdots \times \prod_{i \in I} G_{i} / \mathcal{u}_{n-1}$, so $\pi f$ factors through the projection to that product; and conversely, if $\pi f$ factors through the projection to such a product, then $\mathscr{F}$ is the intersection of some subset of the $U_{k}$ (the minimal set of $u_{k}$ allowing such a factorization). In this connection, we recall

(4) [Bergman 2014, Lemma 1.3, (3) $\Longleftrightarrow(5)]$ A filter $\mathscr{F}$ on a set I can be written as the intersection of finitely many ultrafilters on $I$ if and only if for every partition 
of I into countably many sets $J_{m}(m \in \omega)$, there is at least one $m \in \omega$ such that $I-J_{m} \in \mathscr{F}$.

Here and below, we make the conventions that a partition may include one or more instances of the empty set, and that the intersection of the empty family of filters on a set is the set of all subsets of that set, i.e., the improper filter. (These conventions are needed to make various statements correct in degenerate cases.)

Let us note what (4) tells us about homomorphisms on direct product groups.

Lemma 1. Let $f: \prod_{i \in I} G_{i} \rightarrow B$ be a homomorphism from a direct product of groups $G_{i}$ to a group $B$, which is surjective; or more generally, such that the composite $\pi f: \prod_{i \in I} G_{i} \rightarrow B \rightarrow B / Z(B)$ is surjective. Then the following conditions are equivalent.

(5) $\pi f: \prod_{i \in I} G_{i} \rightarrow B / Z(B)$ does not factor through the natural map $\prod_{i \in I} G_{i} \rightarrow$ $\prod_{i \in I} G_{i} / u_{0} \times \cdots \times \prod_{i \in I} G_{i} / u_{n-1}$ for any finite family $\boldsymbol{u}_{0}, \ldots, u_{n-1}$ of ultrafilters on $I$.

(6) There exists a partition of I into countably many subsets $J_{0}, J_{1}, \ldots$, such that each subgroup $\prod_{i \in J_{n}} G_{i} \subseteq \prod_{i \in I} G_{i}$ contains a pair of elements $x_{n}, y_{n}$ whose images in $B$ under $f$ do not commute.

Proof. The easy direction is $(6) \Longrightarrow(5)$. The fact that $f\left(x_{n}\right)$ and $f\left(y_{n}\right)$ do not commute tells us, in particular, that $f\left(x_{n}\right) \notin Z(B)$. Hence for $\mathscr{F}$ defined by (2) (noting in particular the last line thereof), $I-J_{n} \notin \mathscr{F}$. Since this is true for each $n$, (4) tells us that the filter $\mathscr{F}$ is not a finite intersection of ultrafilters, giving (5).

To get the converse, note that if (5) holds, equivalently, if $\mathscr{F}$ is not a finite intersection of ultrafilters, then by (4) we can partition $I$ into subsets $J_{0}, J_{1}, \ldots$, none of whose complements lies in $\mathscr{F}$; i.e., by the last line of (2), such that each $\prod_{i \in J_{n}} G_{i}$ contains an element $x_{n}$ which is mapped by $f$ to a noncentral element of $B$. Fixing $n$, this says that there exists an element $b \in B$ which does not commute with $f\left(x_{n}\right)$. I claim we can take such a $b$ to be the image of an element $y \in \prod_{i \in I} G_{i}$ under $f$. Indeed, if $f$ is surjective, this is immediate. If instead we have the weaker hypothesis that $\pi f: \prod_{i \in I} G_{i} \rightarrow B \rightarrow B / Z(B)$ is surjective, then we can choose $y \in \prod_{i \in I} G_{i}$ whose image under $f$ is congruent to $b$ modulo $Z(B)$. Since multiplication by an element of $Z(B)$ does not affect what members of $B$ an element commutes with, $f(y)$ does not commute with $f\left(x_{n}\right)$.

Let us now write $y=y_{n} y^{\prime}$, where $y_{n} \in \prod_{i \in J_{n}} G_{i}$ while $y^{\prime} \in \prod_{i \in I-J_{n}} G_{i}$. Then $y^{\prime}$ commutes with $x_{n}$, since they have disjoint supports in our product group. Hence $f\left(y^{\prime}\right)$ commutes with $f\left(x_{n}\right)$; hence if $f\left(y_{n}\right)$ also commuted with $f\left(x_{n}\right)$, then $f(y)=f\left(y_{n}\right) f\left(y^{\prime}\right)$ would commute with $f\left(x_{n}\right)$, contradicting our choice of $y$. Hence, rather, $x_{n}, y_{n} \in \prod_{i \in J_{n}} G_{i}$ have images in $B$ which do not commute, giving (6). 
We can now get the first of our results showing that any group $B$ admitting a map $f$ satisfying (5) must be "big".

Theorem 2. Let $B$ be a group such that there exist a family of groups $\left(G_{i}\right)_{i \in I}$, and a group homomorphism $f: \prod_{i \in I} G_{i} \rightarrow B$, for which the induced homomorphism $\pi f: \prod_{i \in I} G_{i} \rightarrow B / Z(B)$ does not factor through the projection of $\prod_{i \in I} G_{i}$ to the product of finitely many ultraproducts of the $G_{i}$. Then $B$ contains families of elements $\left(a_{S}\right)_{S \subseteq \omega},\left(b_{S}\right)_{S \subseteq \omega}$, indexed by the subsets $S$ of $\omega$, such that:

(7) All the elements $a_{S}(S \subseteq \omega)$ commute with one another, and all the elements $b_{S}$ $(S \subseteq \omega)$ likewise commute with one another.

(8) For $S$ and $T$ disjoint subsets of $\omega$, one has $a_{S} a_{T}=a_{S \cup T}, b_{S} b_{T}=b_{S \cup T}$, and $a_{S} b_{T}=b_{T} a_{S}$.

(9) For subsets $S$ and $T$ of $\omega$ with $\operatorname{card}(S \cap T)=1, a_{S} b_{T} \neq b_{T} a_{S}$.

Proof. Given $G_{i}$ and $f$ as in the hypothesis, i.e., satisfying (5), Lemma 1 gives us sets $J_{n} \subseteq I$ and elements $x_{n}, y_{n}(n \in \omega)$ as in (6). Let $H_{n}=\prod_{i \in J_{n}} G_{i} \subseteq \prod_{i \in I} G_{i}$ $(n \in \omega)$, so that we can regard $\prod_{i \in I} G_{i}$ as $\prod_{n \in \omega} H_{n}$, the $x_{n}$ and $y_{n}$ as elements of that group with singleton supports, and $f$ as a homomorphism $\prod_{n \in \omega} H_{n} \rightarrow B$.

For each subset $S \subseteq \omega$, let $x_{S}$ be the element of $\prod_{n \in \omega} H_{n}$ whose component at $n$ is $x_{n}$ if $n \in S$, and $e$ otherwise, and let elements $y_{S}$ be obtained similarly from the $y_{n}$. It is easy to see that any two elements $x_{S}$ and $x_{T}$ commute with one another in $\prod_{n \in \omega} H_{n}$, and similarly for the $y$ 's; and that for $S$ and $T$ disjoint, $x_{S} x_{T}=x_{S \cup T}$, $y_{S} y_{T}=y_{S} \cup T$, and $x_{S} y_{T}=y_{T} x_{S}$. Hence, letting $a_{S}=f\left(x_{S}\right), b_{S}=f\left(y_{S}\right)$, we get (7) and (8).

For general $S$ and $T$, the commutator $\left[x_{S}, y_{T}\right]$ will have $n$-th component $\left[x_{n}, y_{n}\right]$ if $n \in S \cap T$, and $e$ otherwise. So if $S \cap T$ is exactly $\{n\}$ for some $n \in \omega$, then $f\left(\left[x_{S}, y_{T}\right]\right)=f\left(\left[x_{n}, y_{n}\right]\right)$, which by choice of $x_{n}$ and $y_{n}$ is not $e$, giving (9).

By restricting the elements $b_{T}$ that we consider, we can get a clearer view of the behavior of the elements $a_{S}$ :

Corollary 3. In the situation of Theorem 2, an element $a_{S}(S \subseteq \omega)$ commutes with an element $b_{\{n\}}(n \in \omega)$ if and only if $n \notin S$. Thus, the elements $a_{S}$ exhibit all possible combinations of which members of the countable set $\left\{b_{\{n\}} \mid n \in \omega\right\}$ they commute with. Hence they are distinct modulo $Z(B)$; so their images in $B / Z(B)$ generate a commutative subgroup of continuum cardinality.

Proof. The first sentence is immediate from (8) and (9), and clearly implies the second. Since multiplication by a member of $Z(B)$ does not affect what elements a member of $B$ commutes with, elements which can be distinguished by the latter properties are necessarily distinct modulo $Z(B)$. The group generated by the $a_{S}$ is commutative in view of (7), hence so is the image of that group in $B / Z(B)$. 
Above we have obtained "element-theoretic" consequences of the existence of a map $\prod_{i \in I} A_{i} \rightarrow B$ that does not factor through finitely many ultrafilters. There are also "subgroup-theoretic" consequences. We shall find it convenient to state some of these, not in terms of image subgroups $\pi f\left(\prod_{i \in S} G_{i}\right) \subseteq B / Z(B)$, but in terms of the inverse images $f\left(\prod_{i \in S} G_{i}\right) Z(B)$ of those subgroups in $B$. Let us start by noting some general properties of this construction, independent of whether $\pi f$ factors through finitely many ultraproducts.

Lemma 4. Let $B$ be a group, $\left(G_{i}\right)_{i \in I}$ a family of groups, and $f: \prod_{i \in I} G_{i} \rightarrow B$ a homomorphism which is surjective (or more generally, satisfies $B=f\left(\prod_{i \in I} G_{i}\right) Z(B)$ ). For every subset $S \subseteq I$, let

(10) $B_{S}=f\left(\prod_{i \in S} G_{i}\right) Z(B)$, a normal subgroup of $B$.

Then:

(11) $B_{\varnothing}=Z(B), B_{I}=B$, and for $S, T \subseteq I$, one has $B_{S} B_{T}=B_{S \cup T}$ and $B_{S} \cap B_{T}=$ $B_{S \cap T}$.

(12) For $S, T \subseteq I$, the centralizer of $B_{T}$ in $B_{S}$ is $B_{S-T}$.

Hence (again writing $\pi: B \rightarrow B / Z(B)$ for the quotient map),

(13) For disjoint subsets $S, T \subseteq I, \pi\left(B_{S \cup T}\right)$ is the direct product of its subgroups $\pi\left(B_{S}\right)$ and $\pi\left(B_{T}\right)$.

\section{Moreover,}

(14) If $\left(S_{k}\right)_{k \in K}$ is a family of pairwise disjoint subsets of I, and we let $S=\bigcup_{k \in K} S_{k}$, then the map $\pi\left(B_{S}\right) \rightarrow \prod_{k \in K} \pi\left(B_{S_{k}}\right)$ determined by the projections $\pi\left(B_{S}\right) \rightarrow$ $\pi\left(B_{S_{k}}\right)$ (which by (13) is an isomorphism if $K$ is finite) is always surjective.

Proof. That each $B_{S}$ is normal in $B$, as asserted in (10), follows from the normality of $\prod_{i \in S} G_{i}$ in $\prod_{i \in I} G_{i}$, and the centrality of $Z(B)$ in $B$.

The first three equalities of (11) are immediate, as is the direction $B_{S} \cap B_{T} \supseteq B_{S \cap T}$ of the final equality. Before proving the reverse inclusion, let us note a case of (12) which is also immediate:

(15) If $S$ and $T$ are disjoint subsets of $I$, then $B_{S}$ and $B_{T}$ centralize one another.

To get the remaining part of (11), $B_{S} \cap B_{T} \subseteq B_{S \cap T}$, consider an element of the left-hand side, which we may write

(16) $f(u) z_{1}=f(v) z_{2}, \quad$ where $u \in \prod_{i \in S} G_{i}, v \in \prod_{i \in T} G_{i}$, and $z_{1}, z_{2} \in Z(B)$.

Let us write $u=u^{\prime} u^{\prime \prime}$, where $u^{\prime} \in \prod_{i \in S \cap T} G_{i}$ and $u^{\prime \prime} \in \prod_{i \in S-T} G_{i}$. Thus our element (16) becomes $f\left(u^{\prime}\right) f\left(u^{\prime \prime}\right) z_{1}$. Since $u^{\prime} \in \prod_{i \in S \cap T} G_{i}$, if we can show that $f\left(u^{\prime \prime}\right) \in Z(B)$, then (16) will lie in $f\left(\prod_{i \in S \cap T} G_{i}\right) Z(B)=B_{S \cap T}$, as required. 
Thus, we need to show that $f\left(u^{\prime \prime}\right)$ centralizes $B=B_{I}=B_{S-T} B_{I-(S-T)}$. Since $f\left(u^{\prime \prime}\right) \in B_{S-T}$, it certainly centralizes $B_{I-(S-T)}$. On the other hand if we write the equation in (16) as

$$
f\left(u^{\prime}\right) f\left(u^{\prime \prime}\right) z_{1}=f(v) z_{2}, \quad \text { equivalently, } \quad f\left(u^{\prime \prime}\right)=f\left(u^{\prime}\right)^{-1} f(v) z_{2} z_{1}^{-1},
$$

we see that all the factors on the right lie in $B_{T}$, hence centralize $B_{S-T}$. Hence so does $f\left(u^{\prime \prime}\right)$, completing the proof of the last assertion of (11).

We can now easily prove (12). By (11), $B_{S-T}$ is contained in $B_{S}$, and by (15), it centralizes $B_{T}$, so we need only show that conversely, any element of $B_{S}$ that centralizes $B_{T}$ lies in $B_{S-T}$. As in the preceding argument, we can write our element of $B_{S}$ as $f\left(u^{\prime}\right) f\left(u^{\prime \prime}\right) z$, where $u^{\prime} \in \prod_{i \in S \cap T} G_{i}$ and $u^{\prime \prime} \in \prod_{i \in S-T} G_{i}$. This time, we need to prove that $f\left(u^{\prime}\right) \in Z(B)$. Now since $f\left(u^{\prime}\right) f\left(u^{\prime \prime}\right) z$ centralizes $B_{T}$, and $f\left(u^{\prime \prime}\right)$ and $z$ automatically do, we see that $f\left(u^{\prime}\right)$ centralizes $B_{T}$. Also, since $f\left(u^{\prime}\right) \in B_{S \cap T}$, and $S \cap T$ is disjoint from $I-T, f\left(u^{\prime}\right)$ centralizes $B_{I-T}$. Hence it centralizes $B_{T} B_{I-T}=B$, so it lies in $Z(B)$, as claimed.

The conclusion (13) follows easily from (12) and (11).

To establish (14), we take an element of $\prod_{k \in K} \pi\left(B_{S_{k}}\right)$, lift its component in each $\pi\left(B_{S_{k}}\right)$ to an element of $\prod_{i \in S_{k}} G_{i}$, and regard these together as giving an element of $\prod_{i \in S} G_{i}$; note that the image of this element in $\pi\left(B_{S}\right)$ has the desired property.

Note that in the situation of the above lemma, the subgroups $B_{S}$ need not be distinct for distinct $S \subseteq I$. For instance, if we take a family $\left(G_{i}\right)_{i \in I}$ of noncommutative groups and an ultrafilter $U$ on $I$, let $B=\prod_{i \in I} G_{i} / U$, and let $f: \prod_{i \in I} G_{i} \rightarrow B$ be the quotient map, then the above construction gives only two distinct subgroups of $B: B_{S}=B$ if $S \in \mathcal{U}$, and $B_{S}=Z(B)$ otherwise.

We shall now get a factorization-through-ultraproducts result from the above lemma. Let us (following [Bergman 2014, §4.3]) call subgroups $B^{\prime}, B^{\prime \prime}$ of a group $B$ almost direct factors if $B=B^{\prime} B^{\prime \prime}$, and each of $B^{\prime}, B^{\prime \prime}$ is the centralizer in $B$ of the other. A subgroup $B^{\prime} \subseteq B$ belonging to such a pair (equivalently, such that $B^{\prime}$ is its own double centralizer in $B$, and $B$ is the product of $B^{\prime}$ and its centralizer) will thus be called an almost direct factor of $B$. We shall say $B$ has chain condition on almost direct factors if the partially ordered set of almost direct factors of $B$ has ascending chain condition, equivalently (since that partially ordered set is self-dual under the operation of taking centralizers), if it has descending chain condition. (As noted in [ibid.], these are the analogs for groups of definitions first made for algebras in [Bergman and Nahlus 2011, §6].)

Observe that in the situation treated in Lemma 4, statements (12) and (11) show that for every $S \subseteq I$, the subgroups $B_{S}, B_{I-S}$ are a pair of almost direct factors of $B$. We deduce: 
Theorem 5 (cf. [Bergman 2014, Proposition 4.1]). Let B be a group, and suppose that there exist a family of groups $\left(G_{i}\right)_{i \in I}$ and a homomorphism $f: \prod_{i \in I} G_{i} \rightarrow B$ such that the induced homomorphism $\pi f: \prod_{i \in I} G_{i} \rightarrow B / Z(B)$ is surjective and does not factor through the natural projection of $\prod_{i \in I} G_{i}$ to any finite product of ultraproducts of the $G_{i}$.

Then $B$ does not have chain condition on almost direct factors. In fact, it has a family of almost direct factors order-isomorphic to the lattice $2^{\omega}$, and forming a sublattice of the lattice of subgroups of $B$.

Proof. Given $\left(G_{i}\right)_{i \in I}$ with the indicated non-factorization property, let $J_{0}, J_{1}, \ldots$ be as in Lemma 1. To every subset $S$ of $\omega$, let us associate the subgroup $B_{\cup_{n \in S}} J_{n}$. From Lemma 4 we see that each of these subgroups is an almost direct factor of $B$, and that the lattice relations among the subsets of $\omega$ are also satisfied by the corresponding subgroups; so it will suffice to show that non-inclusions of subsets of $\omega$ yield non-inclusions of subgroups. If $S \nsubseteq T$, take $m \in S-T$. By our assumption on the $J_{n}$, the subgroup $B_{J_{m}}$ is not self-centralizing, hence though it centralizes $B_{\cup_{n \in T} J_{n}}$, it does not centralize $B_{\cup_{n \in S} J_{n}}$; so the latter is not contained in the former.

Neither of the conclusions of Theorem 2 and Theorem 5 implies the other. To get examples of these non-implications, let $G$ be a simple group.

If we embed $G^{\omega}$ in any simple overgroup $B$, then $B$ inherits from $G^{\omega}$ families of elements $a_{S}, b_{S}$ as in Theorem 2; but being simple, $B$ has no nontrivial almost direct decompositions, hence it satisfies chain condition on almost direct factors, i.e., fails to satisfy the conclusion of Theorem 5 .

On the other hand, if we take for $B$ the group $\bigoplus_{\omega} G$ of elements of $G^{\omega}$ having finite support, and let $B_{S}=\bigoplus_{S} G$ for each $S \subseteq \omega$, we find that these subgroups satisfy (11)-(13), hence constitute a system of almost direct factors lattice-isomorphic to $2^{\omega}$, as in Theorem 5. But if $G$ is countable, $B$ will also be so, so it cannot satisfy the conclusion of Theorem 2 .

So neither of these groups $B$ admits a surjective homomorphism $f$ from a direct product group such that $\pi f$ (which in both cases would be $f$, since $Z(B)$ is trivial) fails to factor through finitely many ultraproducts. However, in the first case, only Theorem 5 rules this out, while in the second, only Theorem 2 does.

Though the above example with $B=\bigoplus_{\omega} G$ satisfies (11)-(13), it does not satisfy (14), as can be seen by taking for the $S_{k}$ the singleton subsets of $\omega$. One may ask whether for any group $B$, every system of subgroups $B_{S}(S \subseteq I)$ of $B$ that satisfies all of (11)-(14) arises as in Lemma 4.

The answer is still negative. For instance, suppose $B$ is a group which has trivial center, and which cannot be written as a homomorphic image of a nonprincipal ultraproduct of a family of groups indexed by $\omega$. (We shall see in Section 4 that the 
free group on two generators, among many others, cannot be so written.) Suppose we take a nonprincipal ultrafilter $\mathcal{U}$ on $\omega$, and define $B_{S} \subseteq B$ to be all of $B$ whenever $S \in \mathcal{U}$, and $\{e\}$ otherwise. It is not hard to verify that this family satisfies (11)-(14), but that if it arose as in Lemma 4 (with $\omega$ for $I$ ), then $B$ would be a homomorphic image of $\prod_{n \in \omega} G_{n} /$, contradicting our choice of $B$.

We record a special case of Theorem 5 for easy application to some later examples.

Corollary 6 (to Theorem 5 and its proof). Suppose B is a group with trivial center, and having no nontrivial direct product decomposition. Then every homomorphism from a direct product group $\prod_{i \in I} G_{i}$ onto B factors through a single ultraproduct $\prod_{i \in I} G_{i} /$ U of the $G_{i}$.

\section{Further examples}

Theorem 5 shows that a group $B$ which admits a surjective homomorphism from an infinite direct product group that does not factor through finitely many ultraproducts looks, itself, in some ways, like an infinite direct product - at least after we divide out $Z(B)$. The next example shows that this behavior of $B / Z(B)$ can coexist with very un-product-like behavior in $Z(B)$.

Example 7. Groups $B$ and $G$ and a homomorphism $f: G^{\omega} \rightarrow B$ such that the induced subgroups $B_{S}(S \subseteq \omega)$ are all distinct, but such that the center of each of the given copies of $G$ in $G^{\omega}$ is mapped isomorphically to $Z(B) \neq\{e\}$; and which also show that in (9), the hypothesis $\operatorname{card}(S \cap T)=1$ cannot be weakened to merely say that $S \cap T$ is nonempty and finite.

Construction and proof. Let $k$ be a field, and $G$ the Heisenberg group over $k$; that is, the multiplicative group of upper triangular $3 \times 3$ matrices with 1 's on the main diagonal; equivalently, the group of 3-tuples of elements of $k$ under the multiplication $\left(a, a^{\prime}, a^{\prime \prime}\right)\left(b, b^{\prime}, b^{\prime \prime}\right)=\left(a+b, a^{\prime}+b^{\prime}, a^{\prime \prime}+b^{\prime \prime}+a^{\prime} b\right)$. Clearly, the countable power group $G^{\omega}$ can be described as the group of 3-tuples of elements of the power ring $k^{\omega}$ under the operation given by the same formula.

Let us now take the $k$-vector-space homomorphism $s: \bigoplus_{\omega} k \rightarrow k$ which for each $n$ acts on the $n$-th direct summand by $1 \mapsto s_{n}$, for some specified elements $s_{n} \in k-\{0\}$, and by linear algebra, let us extend $s$ to a vector-space homomorphism $\sigma: k^{\omega} \rightarrow k$. Let $B$ be the homomorphic image of $G^{\omega}$ gotten by dividing $Z\left(G^{\omega}\right)=k^{\omega}$ by $\operatorname{ker}(\sigma)$. This can be described as

$k^{\omega} \times k^{\omega} \times k, \quad$ under the operation

$$
\left(a, a^{\prime}, a^{\prime \prime}\right)\left(b, b^{\prime}, b^{\prime \prime}\right)=\left(a+b, a^{\prime}+b^{\prime}, a^{\prime \prime}+b^{\prime \prime}+\sigma\left(a^{\prime} b\right)\right) .
$$

I claim that $Z(B)=\{0\} \times\{0\} \times k$. To see this, let us first show that every $(a, b, c) \in B$ with $a \neq 0$ is noncentral. Choose $n$ such that $a$ has $n$-th component $a_{n} \neq 0$, and take $b^{\prime} \in k^{\omega}$ to have 1 in the $n$-th position and 0 in all others. Then we 
find that the commutator of $(a, b, c)$ and $\left(0, b^{\prime}, 0\right)$ is $\left(0,0, s_{n} a_{n}\right) \neq e$. The analogous argument shows $(a, b, c)$ noncentral if $b \neq 0$. Elements $(0,0, c)$ are clearly central, so we get the asserted description of $Z(B)$, and we see that this is the image in $B$ of the center of each of our copies of $G$ in $G^{\omega}$, and indeed, of the center of $G^{S} \subseteq G^{\omega}$ whenever $\varnothing \neq S \subseteq \omega$.

So though the images in $B / Z(B)$ of these subgroups $G^{S}$ are the corresponding factors $(k \times k)^{S} \subseteq(k \times k)^{\omega}$, when we look at the images in $Z(B)$ of their centers, the distinctions among them disappear.

To get the final assertion of this example, let us partition $\omega$ into the singletons $J_{n}=\{n\}$, so that in the notation of the proof of Theorem 2, each $H_{n}$ is $G$. For each $n$, let $x_{n}=(1,0,0), y_{n}=(0,1,0)$ in $H_{n}$, and let us use these to construct elements $a_{S}, b_{T} \in B$ as in that proof. Then if $S$ and $T$ are subsets of $\omega$ which intersect in a finite set $\left\{n_{0}, \ldots, n_{d-1}\right\}$, we see that in $B$ the commutator $\left[a_{S}, b_{T}\right]$ is $\left(0,0, s_{n_{0}}+\cdots+s_{n_{d-1}}\right)$. If $d=1$ this is necessarily a nonidentity element, as stated in (9); but if $S$ and $T$ intersect in more than one element, this may or may not be true, depending on the choice of the $s_{n}$. (In particular, if the field $k$ is finite, then whatever the $s_{n}$, there must be some nonempty family of $\leq \operatorname{card}(k) s_{n}$ 's that sum to zero.) So the restriction $\operatorname{card}(S \cap T)=1$ in (9) cannot be dropped.

In the above example, the focus was on the part of the map going into $Z(B)$; the map $G^{\omega} \rightarrow B / Z(B)$ was a straightforward homomorphism of direct products. But this is not always the case; that is, the maps which (14) shows to be surjective need not, in general, be isomorphisms. For instance, in the example mentioned immediately after the proof of Lemma 4 , where the $G_{i}$ were arbitrary noncommutative groups, and $f$ was the map $\prod_{i \in I} G_{i} \rightarrow\left(\prod_{i \in I} G_{i}\right) / \mathcal{U}$, for $U$ an ultrafilter on $I$, if $\mathcal{U}$ is nonprincipal and we take for the $S_{k}$ all the singletons $\{i\}(i \in I)$, so that $S=I$, then each $\pi\left(B_{S_{k}}\right)$ is trivial, but $\pi\left(B_{S}\right)$ is not.

One can, of course, modify this example to get one which also has the property that every $G_{i}$ has nontrivial image in $B / Z(B)$ :

Example 8. A group homomorphism $\prod_{n \in \omega} G_{n} \rightarrow B$ where all the $B_{\{n\}} / Z(B)$ are nonzero (so that all the $B_{S}$ are distinct), but not all the surjections of (14) are isomorphisms.

Construction. Let $G_{n}(n \in \omega)$ be groups with trivial centers, each having a proper nontrivial normal subgroup $N_{n} \triangleleft G_{n}$ such that $G_{n} / N_{n}$ also has trivial center. Let $\mathcal{U}$ be any nonprincipal ultrafilter on $\omega$, let $H=\left(\prod_{n \in \omega} G_{n}\right) / \mathcal{U}$, and let $f: \prod_{n \in \omega} G_{n} \rightarrow H \times \prod_{n \in \omega} G_{n} / N_{n}$ be the map obtained from the obvious homomorphisms $\prod_{n \in \omega} G_{n} \rightarrow H$ and $\prod_{n \in \omega} G_{n} \rightarrow \prod_{n \in \omega} G_{n} / N_{n}$. Let $B$ be the image of $f$.

For $S \subseteq \omega$, what does $B_{S}$ look like? This depends on whether or not $S \in \mathcal{U}$. If not, we see that $B_{S}=\prod_{n \in S} G_{n} / N_{n}$; in particular, for every $n \in \omega$ we have $B_{\{n\}}=G_{n} / N_{n}$. 
Thus for $S \notin U$, the group $B_{S}$ can be identified with $\prod_{n \in S} B_{\{n\}}$. However, when $S \in \mathcal{U}$, the $H$-component of $B_{S}$ will be the full group $H$, which carries structure from the normal subgroups $N_{n}$ which is ignored by each group $B_{\{n\}}$; so in these cases, the natural map $B_{S} \rightarrow \prod_{n \in S} B_{\{n\}}$ is not one-to-one.

One can generalize the above construction by replacing $\mathcal{U}$ with an arbitrary filter $\mathscr{F}$, though the description of the groups $B_{S}$ is more complicated to state when $S$ is neither a member of $\mathscr{F}$ nor the complement of one. And, of course, one can set up examples based on more than one system of normal subgroups and more than one filter.

In the above example, though the system of subgroups $B_{S}$ described does not have the property that the maps of (14) are isomorphisms, the group $B$ has other systems of subgroups that can be shown to have that property. Here is an example having no such family.

Example 9. A group $B$ having elements $a_{S}$ and $b_{S}(S \subseteq \omega)$ satisfying (7)-(9), and distinct subgroups $B_{S}(S \subseteq \omega)$ satisfying (10)-(13), but having no such system of distinct subgroups also satisfying (14) for any infinite family of disjoint nonempty sets $\left(s_{k}\right)_{k \in K}$; so that B cannot admit a surjective homomorphism from a direct product group which does not factor through the product of finitely many ultraproducts.

Construction and sketch of proof. Let $G$ be an infinite simple group, and $B$ the subgroup of $G^{\omega}$ consisting of those $\omega$-tuples assuming only finitely many distinct values in $G$. If we choose a pair of noncommuting elements $x, y \in G$, and for each $n \in \omega$ let $x_{n}$ be the element $x$ of the $n$-th copy of $G$, and $y_{n}$ the element $y$ thereof, then we see that the elements $x_{S}$ and $y_{S}(S \subseteq \omega)$, constructed as in Theorem 2, will lie in $B$, and, renamed $a_{S}$ and $b_{S}$, will satisfy (7)-(9). Similarly, if we let $B_{S}$ be the subgroup of $B$ consisting of elements with support in $S$, then (11)-(13) are immediate.

I will now sketch why $B$ admits no system of nontrivial almost direct factors $B_{S_{k}}$ and $B_{S}$ satisfying (14) for any infinite $K$. Note that $B$ has trivial center, so that almost direct factors are simply direct factors. Now it is easy to verify using the simplicity of $G$ that if $B$ has a direct product decomposition $B=B^{\prime} \times B^{\prime \prime}$, then for each $n \in \omega$, one of $B^{\prime}, B^{\prime \prime}$ has as $n$-th coordinates all members of $G$, while the other has only $e$ in that coordinate. From this one can deduce that every such decomposition has the form $B^{\prime}=B_{S}, B^{\prime \prime}=B_{\omega-S}$ for some $S \subseteq \omega$. We can now combine the "finitely many distinct values" condition in the definition of $B$ with the fact that $G$ is infinite to see the impossibility of an infinite family of nontrivial almost direct factors $B_{S_{k}}$ satisfying the surjectivity condition (14).

Lemma 4 and the method of proof of Theorem 5 now show that every homomorphism from a direct product onto $B$ must factor through finitely many ultraproducts. 
(For some other results on the subgroup of a power group $G^{I}$ consisting of the elements with only finitely many distinct coordinates - though for abelian groups see [Bergman 1972].)

\section{Conditions forcing the ultrafilters to be principal}

We have obtained conditions that force group homomorphisms $\prod_{i \in I} G_{i} \rightarrow B$ to factor through the direct product of finitely many ultraproducts of the $G_{i}$. When can we say that any map that so factors must in fact factor through the product of finitely many $G_{i}$; i.e., that the ultrafilters involved must be principal?

Here set-theoretic considerations come in. If $\kappa$ is a measurable cardinal, then sets $I$ of cardinality $\geq \kappa$ admit nonprincipal $\kappa$-complete ultrafilters; that is, ultrafilters closed under all $<\kappa$-fold intersections. (Two quick terminological notes: (i) The condition of being closed under countable intersections, which by the above definition is $\aleph_{1}$-completeness, is also called countable completeness. (ii) We shall follow the definition of measurable cardinal used in [Chang and Keisler 1990], which counts $\aleph_{0}$ as measurable; so we will write "uncountable measurable cardinal" for what many authors simply call a measurable cardinal.)

If $\kappa$ is an uncountable measurable cardinal and $I$ a set of cardinality $\geq \kappa$, and we take a family $\left(G_{i}\right)_{i \in I}$ of groups (or more generally, of any sort of algebraic structures defined by finitely many finitary operations) whose cardinalities have a common bound $<\kappa$, then their ultraproducts with respect to $\kappa$-complete ultrafilters behave very much as do ordinary ultraproducts of finite groups with a common finite bound on their orders; to wit, every such ultraproduct is isomorphic to one of the $G_{i}$. Hence, if there exists such a cardinal $\kappa$, then every group $B$ of cardinality $<\kappa$ can be represented as an ultrapower of itself with respect to a nonprincipal $\kappa$-complete ultrafilter $U$. So for every such $B$ we get a surjective homomorphism $B^{I} \rightarrow B$ which factors through the ultrapower $B^{I} / U$ but not through finitely many projection maps - which seems to be bad news for the type of result we are hoping for.

However, it is known that if uncountable measurable cardinals exist, they must be quite enormous [Chang and Keisler 1990, Theorem 4.2.14], and that if the standard set theory, ZFC, is consistent, it is consistent with the nonexistence of such cardinals. Hence it would be reasonable to work under the assumption that no uncountable measurable cardinals exist, or, if they exist, to restrict our index sets to cardinalities less than all such cardinals.

The next observation shows that when doing the spade-work of our investigation, we can in fact restrict attention to the case where our index set is countable.

Lemma 10. If $B$ is a group, then the following conditions are equivalent.

(18) $B$ is a homomorphic image of an ultraproduct of a family of groups indexed by an arbitrary set $I$, with respect to some ultrafilter $U$ on I that is not countably 
complete, equivalently, that is not $\kappa$-complete for any uncountable measurable cardinal $\kappa$.

(19) B is a homomorphic image of an ultraproduct of a family of groups indexed by $\omega$, with respect to a nonprincipal ultrafilter on $\omega$.

The same is true with "groups" replaced by objects of any other variety of finitary algebras, in the sense of universal algebra.

Proof. The equivalence referred to in (18) follows from the fact that any countably complete ultrafilter must be $\kappa$-complete for some uncountable measurable cardinal $\kappa$ [Chang and Keisler 1990, Proposition 4.2.7].

Since a nonprincipal ultrafilter on $\omega$ is not countably complete, we have (19) $\Longrightarrow$ (18). On the other hand, it is easy to show that if $U$ is a non-countably-complete ultrafilter on a set $I$, then $I$ can be partitioned as $\bigcup_{n \in \omega} J_{n}$ where no $J_{n}$ belongs to $\mathcal{U}$. In this situation we find that $\left\{S \subseteq \omega \mid \bigcup_{n \in S} J_{n} \in \mathcal{U}\right\}$ is a nonprincipal ultrafilter $U^{\prime}$ on $\omega$, and that given groups $G_{i}(i \in I)$, the natural map $\prod_{i \in I} G_{i} \rightarrow \prod_{i \in I} G_{i} / \mathcal{U}$ factors through $\prod_{n \in \omega}\left(\prod_{i \in J_{n}} G_{i}\right) / \mathcal{U}^{\prime}$. Hence, writing $\prod_{i \in J_{n}} G_{i}=H_{n}$, we see that if, as in (18), $B$ is a homomorphic image of $\prod_{i \in I} G_{i} / \mathcal{U}$, then it is also a homomorphic image of $\prod_{n \in \omega} H_{n} / \mathcal{U}^{\prime}$, giving (19).

The final assertion is clear. (The assumption that our algebras are finitary is needed to insure that algebra structures are induced on ultraproducts of such algebras.)

So below, it will suffice to examine which groups are homomorphic images of nonprincipal ultraproducts of countable families of groups. For brevity, we shall call an ultraproduct of a countable family a "countable ultraproduct".

My first guess was that if $B$ was such a homomorphic image, then the cardinality of $B / Z(B)$ would have to be either finite or at least the cardinality of the continuum. But Tom Scanlon suggested the following counterexample.

Lemma 11 (T. Scanlon, personal communication). Let $B$ be the semidirect product of the additive group $\mathbb{Q}$ of rational numbers, and the 2-element group $\{ \pm 1\}$, determined by the multiplicative action of the latter on the former. (I.e., $B$ has underlying set $\{ \pm 1\} \times \mathbb{Q}$, and multiplication $(\alpha, a)(\beta, b)=(\alpha \beta, \beta a+b)$.)

Then every ultrapower of $B$ admits a homomorphism onto $B$. Hence though $B=B / Z(B)$ is countable, it is a homomorphic image of a nonprincipal countable ultraproduct of groups.

Proof. Clearly, the only elements of $B$ that commute with $(-1,0)$ are those with second component 0 , while the only elements that commute with $(1,1)$ are those with first component 1 ; so $Z(B)=\{e\}$, justifying the formula $B=B / Z(B)$.

It is easy to see that for any ultrafilter $U$ on any index set $I$, the ultrapower $B^{I} / U$ will be the semidirect product of $\{ \pm 1\}$ and $\mathbb{Q}^{I} / \mathcal{U}$ determined by the natural action 
of the former group on the latter. Now $\mathbb{Q}^{I} / \mathcal{U}$, like $\mathbb{Q}$, is a nontrivial torsion-free divisible group, i.e., a nontrivial $\mathbb{Q}$-vector-space, and, as such, admits a surjective homomorphism $\varphi: \mathbb{Q}^{I} / U \rightarrow \mathbb{Q}$. The map $B^{I} / \mathcal{U} \rightarrow B$ given by $(\alpha, \beta) \mapsto(\alpha, \varphi(\beta))$ is easily seen to be a surjective homomorphism, as claimed.

By Corollary 6, every homomorphism from a direct product group $\prod_{i \in I} G_{i}$ onto the above group $B$ factors through a single ultraproduct of the $G_{i}$; but the above result shows that (even when the index set is countable) the ultrafilter involved need not be principal.

In fact, the only condition I know that guarantees factorization through finitely many of the $G_{i}$ is based on requiring appropriate abelian subgroups of $B$ to satisfy similar factorization properties as abelian groups. The key observation is:

Lemma 12. Suppose B is a homomorphic image of a nonprincipal countable ultraproduct of groups, $\left(\prod_{n \in \omega} G_{n}\right) / \mathcal{U}$. Then every element $b \in B$ lies in a homomorphic image within $B$ of $\mathbb{Z}^{\omega} / \mathcal{U}$, a nonprincipal countable ultrapower of $\mathbb{Z}$.

Proof. Given $b \in B$, let $b$ be the image of $\left(g_{n}\right)_{n \in \omega} \in \prod_{n \in \omega} G_{n}$. Then the homomorphism $\gamma: \mathbb{Z}^{\omega} \rightarrow \prod_{n \in \omega} G_{n}$ taking $\left(m_{n}\right)_{n \in \omega}$ to $\left(g_{n}^{m_{n}}\right)_{n \in \omega}$ induces a homomorphism $\gamma^{\prime}: \mathbb{Z}^{\omega} / \mathcal{U} \rightarrow\left(\prod_{n \in \omega} G_{n}\right) / \mathcal{U}$, with which it forms a commuting square. Hence the composite map $\mathbb{Z}^{\omega} \rightarrow \prod_{n \in \omega} G_{n} \rightarrow \prod_{n \in \omega} G_{n} / \mathcal{U} \rightarrow B$, which carries $(1,1, \ldots) \in \mathbb{Z}^{\omega}$ to $b$, factors through $\mathbb{Z}^{\omega} / \mathcal{U}$; so $b$ lies in a homomorphic image of that group.

To see that this puts strong restrictions on groups $B$ admitting such homomorphisms, note that every slender abelian group, in particular, the infinite cyclic group, has the property of not being a homomorphic image of a nonprincipal countable ultrapower of $\mathbb{Z}$. We will see wider classes of abelian groups with this property in the next section.

Though this note emphasizes the separate conditions that maps from infinite products yield factorizations through finitely many ultraproducts, and that the ultraproducts in all such factorizations are principal, let us record how the above lemma allows one to combine results of the former sort obtained in Section 2 above, and results of the latter sort for abelian groups, which will be obtained in Sections 5-6, to give sufficient conditions for all maps from a direct product of groups to factor through finitely many projection maps.

Theorem 13. Let $B$ be a group with the property that for every homomorphism from a direct product group,

$$
\begin{aligned}
& f: \prod_{i \in I} G_{i} \rightarrow B \text { such that the composite homomorphism } \pi f: \prod_{i \in I} G_{i} \rightarrow B / Z(B) \\
& \text { is surjective, }
\end{aligned}
$$

the map $\pi f$ factors through the projection to finitely many ultraproducts of the $G_{i}$ (cf. Section 2 above). 
Suppose, moreover, that for every almost direct factor $B^{\prime} \neq Z(B)$ of $B$, the group $B^{\prime} / Z(B)$ contains at least one element $b$ which does not lie in any homomorphic image therein of a nonprincipal countable ultraproduct of copies of $\mathbb{Z}$ (cf. Sections 5-7 below).

Then for every homomorphism (20) such that card(I) is less than every uncountable measurable cardinal (if any such cardinals exist), the composite $\pi f$ : $\prod_{i \in I} G_{i} \rightarrow B / Z(B)$ factors through the product of finitely many of the $G_{i}$.

Proof. Given a homomorphism (20) satisfying the indicated bound on card( $I$ ), let us factor $\pi f$ through a direct product $\prod_{i \in I} G_{i} / \mathcal{U}_{0} \times \cdots \times \prod_{i \in I} G_{i} / \mathcal{u}_{m-1}$, where $U_{0}, \ldots, U_{m-1}$ are distinct ultrafilters on $I$. Without loss of generality, we may assume that each $\prod_{i \in I} G_{i} / \mathcal{U}_{k}$ has nontrivial image in $B / Z(B)$. Choosing a partition $I=J_{0} \cup \cdots \cup J_{m-1}$ with $J_{k} \in \mathcal{U}_{k}$, we get, by Lemma 4 , an almost direct decomposition of $B$ into subgroups $B_{J_{k}}$. Now suppose one of our ultrafilters $\boldsymbol{U}_{k}$ were not principal. By our assumption on the cardinality of $I, U_{k}$ is not $\kappa$-complete for any uncountable measurable cardinal $\kappa$, hence by Lemma $10,(18) \Longrightarrow(19)$, $B_{J_{k}} / Z(B)$ satisfies the hypothesis of Lemma 12 . But since $B_{J_{k}}$ is an almost direct factor of $B$, by assumption $B_{J_{k}} / Z(B)$ has an element $b$ whose properties contradict the conclusion of that lemma. So, rather, every $\varkappa_{k}$ must be principal, say generated by a singleton $\left\{n_{k}\right\} \subseteq J_{k}$. Hence our factorization through

$$
\left(\prod_{i \in I} G_{i}\right) / u_{0} \times \cdots \times\left(\prod_{i \in I} G_{i}\right) / u_{m-1}
$$

is in fact a factorization through $G_{n_{0}} \times \cdots \times G_{n_{m-1}}$.

Quick examples of groups $B$ to which the above result applies are free groups on more than one generator, and the infinite dihedral group. Indeed, since both groups have trivial center, the " $/ Z(B)$ " in the statement can be ignored, and since neither has a nontrivial direct product decomposition, it suffices to verify that each has an element $b$ not contained in any homomorphic image of a nonprincipal countable ultraproduct of copies of $\mathbb{Z}$. In a free group, every nontrivial abelian subgroup is infinite cyclic, hence slender, so any nonidentity element can serve as such a $b$. In the dihedral group $D=\left\langle x, y \mid x^{2}=e=y^{2}\right\rangle$, the element $b=x y$ generates an infinite cyclic subgroup which is its own centralizer, again establishing the hypothesis of the theorem. Another class of examples is noted in:

Corollary 14 (to Lemma 12). Let $X$ be an infinite set, and $B$ a group of permutations of $X$ having a cyclic subgroup $\langle b\rangle$ whose action on $X$ has exactly one infinite orbit (no restriction being assumed on the number of finite orbits of $\langle b\rangle$ ). Then the centralizer of $b$ in $B$ admits a homomorphism to $\mathbb{Z}$ taking $b$ to 1 . Hence $B$ is not $a$ homomorphic image of a nonprincipal ultraproduct of a countable family of groups. 
In particular, this is true if $B$ is the full symmetric group on $X$, or more generally, if for some filter $\mathscr{F}$ on $X$ not consisting entirely of cofinite subsets, $B$ is the group of permutations of $X$ whose fixed sets belong to $\mathscr{F}$.

Proof. If two permutations $a$ and $b$ of a set $X$ commute, it is easy to see that $a$ will carry orbits of $\langle b\rangle$ to orbits of $\langle b\rangle$, and, of course, the image orbits will have the same cardinalities as the original orbits. Hence, if $\langle b\rangle$ has a unique infinite orbit $Y$, then $a$ must carry $Y$ to itself; and it is easy to verify that it must act on $Y$ by some power $b^{n_{a}}$ of $b$. The function $a \mapsto n_{a}$ now gives the desired homomorphism of the centralizer of $b$ onto $\mathbb{Z}$. Hence, every commutative subgroup of $B$ containing $b$ admits a homomorphism onto $\mathbb{Z}$, so as in the other examples discussed above, $B$ is not a homomorphic image of a nonprincipal ultraproduct of a countable family of groups.

Now if $\mathscr{F}$ is a filter on $X$ containing a set $W$ which is not cofinite, we can take a countably infinite subset $Y \subseteq X-W$, and let $b$ be a permutation which has $Y$ as an orbit, and fixes all other points of $X$. This gives the final assertion of the corollary. The full symmetric group on $X$ is the particular case where $\mathscr{F}$ is the improper filter on $X$.

(With a little more work, one can get a result similar to the first paragraph of the above corollary under the weaker assumption that $\langle b\rangle$ has at least one but only finitely many distinct infinite orbits, say $\langle b\rangle x_{0}, \ldots,\langle b\rangle x_{d-1} \subseteq X$. In this case, for each $a$ centralizing $b$, we find that $a x_{i}=b^{n_{a, i}} x_{\pi_{a}(i)}(0 \leq i<d)$ for some permutation $\pi_{a}$ of $\{0, \ldots, d-1\}$ and integers $n_{a, 0}, \ldots, n_{a, d-1}$. It is then easy to verify that the map $a \mapsto \sum_{i} n_{a, i}$ is a homomorphism from the centralizer of $b$ to $\mathbb{Z}$, which carries $b$ to $d$.)

The next result, in contrast, gives a large class of groups that $d o$ admit surjective homomorphisms from nonprincipal countable ultraproducts. The construction appears to be well known, but I have not been able to find a reference.

Proposition 15. If a group B admits a compact Hausdorff group topology, then for any set I and any ultrafilter $U$ on I, there exists a homomorphism $B^{I} / \mathcal{U} \rightarrow B$ left-inverse to the natural embedding $B \rightarrow B^{I} \rightarrow B^{I} / \mathcal{U}$ (where the first arrow is the diagonal map).

Hence, every group B admitting a compact Hausdorff group topology is a homomorphic image of a nonprincipal countable ultraproduct of groups; hence so is every homomorphic image of such a group.

These statements hold, more generally, with groups replaced by the objects of any variety of finitary algebras, in the sense of universal algebra.

Sketch of proof. Fix a compact Hausdorff group topology on $B$. Given $x \in B^{I}$, let us associate to each $S \in \mathcal{U}$ the set $X_{S}=\left\{x_{i} \mid i \in S\right\} \subseteq B$. These sets clearly have the finite intersection property, hence so do their closures. On the other hand, with 
the help of the definition of ultrafilter and the Hausdorffness of our topology, it is easy to verify that those closures can have no more than one common point. Hence by compactness, the system of sets $X_{S}$ must converge to a single point of $B$. It is immediate that the map associating to $x$ the limit point of this system depends only on the image of $x$ in $B^{I} / \mathcal{U}$, and so induces a map $B^{I} / U \rightarrow B$, and it is easy to verify that this is a homomorphism with the asserted properties.

The statements in the second paragraph of the lemma clearly follow. The final generalization holds by the same reasoning.

By the above result, such a $B$ is a homomorphic image of $B^{I} / \mathcal{U}$ for every ultrafilter $U$ on every set $I$. This suggests the following question, where for simplicity we limit ourselves to $I=\omega$.

Question 16. If $\mathcal{U}, \mathcal{U}^{\prime}$ are nonprincipal ultrafilters on $\omega$, can every group $B$ which can be written as a homomorphic image of an ultraproduct of groups with respect to $U$ also be written as a homomorphic image of an ultraproduct of groups with respect to $\mathcal{U}^{\prime}$ ?

Question 17. If the answer to Question 16 is negative, is it at least true that for any two ultrafilters $U$ and $U^{\prime}$ on $\omega$, there exists an ultrafilter $U^{\prime \prime}$ on $\omega$ such that every group which can be written as a homomorphic image of an ultraproduct of groups with respect to $U$ or with respect to $U^{\prime}$ can be written as a homomorphic image of an ultraproduct with respect to $\mathcal{U}^{\prime \prime}$ ?

If Question 17 has a positive answer, one can deduce that the class of groups which can be written as homomorphic images of nonprincipal countable ultraproducts of groups is closed under finite direct products.

Proposition 15 also leads one to wonder whether every group $B$ which can be written as a homomorphic image of a nonprincipal countable ultraproduct of groups can in fact be written as a homomorphic image of a nonprincipal countable ultrapower $B^{\omega} / \mathcal{U}$ of itself, via a left inverse to the natural embedding $B \rightarrow B^{\omega} / \mathcal{U}$. The answer is negative; we shall see in the second paragraph after Lemma 28 that there exist abelian groups for which this is not true.

Let us note a couple of groups $B$ for which the results of this section do not, as far as I can see, give us any information.

Question 18. Can either of the following groups be written as a homomorphic image of a nonprincipal ultraproduct of a countable family of groups?

(i) An infinite finitely generated Burnside group?

(ii) The group of those permutations of an infinite set that move only finitely many elements? (Contrast Corollary 14.)

Let us also record, since we know no counterexample, 
Question 19. Is the converse to Lemma 12 true? That is, if $\mathcal{U}$ is an ultrafilter on $\omega$, and $B$ is a group such that every $b \in B$ lies in a homomorphic image within $B$ of $\mathbb{Z}^{\omega} / \mathcal{U}$, must $B$ be a homomorphic image of an ultraproduct group $\prod_{i \in \omega} G_{i} / \mathcal{U}$ ?

A positive answer seems extremely unlikely. It would imply, in particular, that every torsion group was such a homomorphic image for every $\mathcal{u}$. (So it would imply positive answers to both parts of Question 18.)

We remark that the results we have obtained so far show that the two sorts of properties of an object $B$ that we are considering in this note - (a) that surjective homomorphisms from direct products onto $B$ yield factorizations through finitely many ultraproducts, and (b) that when one has such a factorization, and the index set of the product is countable, the ultraproducts involved must be principal - are independent, for groups. Theorem 13 gave us examples satisfying both (a) and (b), such as the free group on more than one generator, and the infinite dihedral group. Any infinite direct product of free groups on more than one generator will still satisfy (b) (since a homomorphism from a nonprincipal countable ultraproduct group into such a product will have trivial composite with the projection onto each factor, hence must be trivial), but will fail to satisfy (a), by virtue of being an infinite direct product. Examples satisfying (a) but not (b) are given by groups satisfying the hypotheses of both Corollary 6 and Proposition 15; for instance, finite simple groups. Finally, infinite direct products of such examples satisfy neither (a) nor (b).

(Incidentally, if a map $\prod_{i \in I} G_{i} \rightarrow B$ factors as

$$
\prod_{i \in I} G_{i} \rightarrow\left(\prod_{i \in I} G_{i}\right) / \mathcal{U}_{0} \times \cdots \times\left(\prod_{i \in I} G_{i}\right) / \mathcal{U}_{m-1} \rightarrow B,
$$

one or more of the factors $\left(\prod_{i \in I} G_{i}\right) / \mathcal{U}_{k}$ may be irrelevant to the factorization, i.e., may map trivially to $B$. In condition (b) in the above discussion, we understand the phrase "the ultraproducts involved" to exclude such "irrelevant" factors; if we did not, (b) could never hold.)

\section{Abelian groups}

We have seen that in the study of homomorphisms on products of nonabelian groups, the analogous questions for abelian groups are important. We now turn to that case.

Although, as just noted, the two sorts of condition we are interested in are independent for nonabelian groups, we shall find that this is not true of the corresponding conditions on abelian groups.

First, some notation, language, and basic observations.

Definition 20. In Sections 5-7, we shall use additive notation in abelian groups.

In groups $\mathbb{Z}^{\omega},(\mathbb{Z} / p \mathbb{Z})^{\omega}$, etc., we shall write $\delta_{n}(n \in \omega)$ for the element having 1 in the $n$-th position and 0 in all other positions. 
An abelian group $B$ is called slender if it is torsion-free, and every homomorphism $f: \mathbb{Z}^{\omega} \rightarrow B$ annihilates all but finitely many of the $\delta_{n}$.

The above definition of a slender abelian group is standard, but the condition that $B$ be torsion-free is redundant: no $B$ with torsion satisfies the condition on homomorphisms. For in such a $B$, we can choose an element $b$ of prime order $p$, define the homomorphism $\bigoplus_{n \in \omega} \mathbb{Z} / p \mathbb{Z} \rightarrow\langle b\rangle$ taking each $\delta_{n}$ to $b$, extend this, by linear algebra over the field $\mathbb{Z} / p \mathbb{Z}$, to a homomorphism $(\mathbb{Z} / p \mathbb{Z})^{\omega} \rightarrow\langle b\rangle$, and precompose with the natural map $\mathbb{Z}^{\omega} \rightarrow(\mathbb{Z} / p \mathbb{Z})^{\omega}$, to get a map $\mathbb{Z}^{\omega} \rightarrow B$ that does not annihilate any $\delta_{n}$.

The condition of slenderness is stronger than it looks. Indeed, our statement of that condition in Section 1 implicitly incorporated the following striking complementary fact.

(21) [Fuchs 1973, fact (f) on p. 159] If $B$ is a slender abelian group, then the only homomorphism $f: \mathbb{Z}^{\omega} \rightarrow B$ which annihilates all the elements $\delta_{n}(n \in \omega)$ is 0 .

We can now prove:

Proposition 21. The following conditions on an abelian group $B$ are equivalent.

(22) There exists a surjective homomorphism $f: \prod_{i \in I} A_{i} \rightarrow B$ from the direct product of a family of abelian groups to $B$, which does not factor through the natural map from $\prod_{i \in I} A_{i}$ to the direct product of finitely many countably complete ultraproducts of the $A_{i}$.

(23) There exists a surjective homomorphism $f: \prod_{n \in \omega} A_{n} \rightarrow B$ from the direct product of a countable family of abelian groups to $B$, which does not factor through the projection of $\prod_{n \in \omega} A_{n}$ to the direct product of finitely many ultraproducts (principal or nonprincipal) of the $A_{n}$.

(24) B is not slender.

These are also equivalent to the variants of conditions (22) and (23) without the assumption that $f$ be surjective.

Proof. We start with the final sentence. Conditions (23) and (22) certainly imply the corresponding statements without the condition of surjectivity. Conversely (as noted in Section 1), if we have an example of either of those conditions minus the surjectivity restriction, we can get one satisfying that condition by passing from the given map $\prod_{i \in I} A_{i} \rightarrow B$ to the obvious surjective map $B \times \prod_{i \in I} A_{i} \rightarrow B$.

Let us now show that (24) $\Longrightarrow$ (23) $\Longrightarrow$ (22) $\Longrightarrow$ (24).

Given (24), take a map $f: \mathbb{Z}^{\omega} \rightarrow B$ witnessing the failure of slenderness, i.e., carrying infinitely many of the $\delta_{n}$ to nonzero values. If $f$ factored through a product of finitely many ultrapowers, $\mathbb{Z}^{\omega} / \mathcal{U}_{0} \times \cdots \times \mathbb{Z}^{\omega} / \boldsymbol{U}_{m-1}$, then the only elements $\delta_{n}$ which could have nonzero image under $f$ would be those such that one of the $u_{k}$ 
was the principal ultrafilter generated by $\{n\}$, of which there can be at most finitely many. So there is no such factorization, so $f$ witnesses (23) (in its version without the hypothesis of surjectivity).

Clearly, (23) $\Longrightarrow(22)$.

Given $f$ as in (22), we shall prove (24) by considering two cases. First suppose that $f$ can be factored through a product of finitely many ultraproducts $\prod_{i \in I} A_{i} / \mathcal{u}_{0} \times \cdots \times \prod_{i \in I} A_{i} / \mathcal{u}_{m-1}$, but that not all the $u_{k}$ can be taken countably complete. Note that $f$ is the sum of homomorphisms $f_{k}(k=0, \ldots, m-1)$ that factor through the respective ultraproducts $\prod_{i \in I} A_{i} / \mathcal{U}_{k}$, and we can drop from this sum, and hence from our factorization, any factors $\prod_{i \in I} A_{i} / \mathcal{u}_{k}$ such that $f_{k}$ is zero. Hence for some $k$ with $u_{k}$ not countably complete, we must have a nonzero homomorphism $f_{k}: \prod_{i \in I} A_{i} / u_{k} \rightarrow B$. The statement that $u_{k}$ is not countably complete is equivalent to saying that there exists a partition $I=J_{0} \cup \cdots \cup J_{n} \cup \cdots$ such that none of the $J_{n}$ lie in $\varkappa_{k}$; in other words, such that for each $n, f_{k} \mid \prod_{i \in J_{n}} A_{i}=0$. If we regard $f_{k}$ as a map $\prod_{n \in \omega}\left(\prod_{i \in J_{n}} A_{i}\right) \rightarrow B$, the fact that it is nonzero means that we can choose an element $\left(x_{n}\right)_{n \in \omega} \in \prod_{n \in \omega}\left(\prod_{i \in J_{n}} A_{i}\right)$ which $f_{k}$ sends to a nonzero element of $B$, though we know that it takes each $x_{n}$ to 0 . Using this element $\left(x_{n}\right)_{n \in \omega}$, let us construct a map $\mathbb{Z}^{\omega} \rightarrow B$ by taking each $\left(d_{n}\right)_{n \in \omega} \in \mathbb{Z}^{\omega}$ to $\left(d_{n} x_{n}\right)_{n \in \omega}$, and applying $f_{k}$ to this $\omega$-tuple. This gives a homomorphism $\mathbb{Z}^{\omega} \rightarrow B$ which is zero on each $\delta_{n}$, but not on $(1, \ldots, 1, \ldots)$. Thus, by $(21), B$ is not slender. (Alternatively, we can get a direct contradiction to the definition of slenderness by choosing $f_{k}$ and $\left(x_{n}\right)_{n \in \omega}$ as above, and mapping $\left(d_{n}\right)_{n \in \omega} \in \mathbb{Z}^{\omega}$ to $f_{k}\left(\left(\sum_{m<n} d_{m}\right) x_{n}\right)_{n \in \omega} \in B$.)

There remains the case where $f$ cannot be factored through any product of finitely many ultraproducts of the $A_{i}$. Then the filter $\mathscr{F}$ of subsets $S \subseteq I$ such that $f$ can be factored through $\prod_{i \in S} A_{i}$ is not a finite intersection of ultrafilters, so by (4) there exists a partition $I=J_{0} \cup \cdots \cup J_{n} \cup \cdots$ such that $I-J_{n} \notin \mathscr{F}$ for all $n$; in other words, such that each $\prod_{i \in J_{n}} A_{i} \subseteq \prod_{i \in I} A_{i}$ has nonzero image under $f$. Choosing an $x_{n}$ in each $\prod_{i \in J_{n}} A_{i}$ with nonzero image, we construct as in the preceding case a homomorphism $\mathbb{Z}^{\omega} \rightarrow B$. This time, that homomorphism will be nonzero on every $\delta_{n}$, showing that $B$ does not satisfy the definition of slenderness.

Slender abelian groups have been precisely characterized [Nunke 1961; Fuchs 1973, Proposition 95.2]: they are the abelian groups which have no torsion elements, and contain no embedded copies of either $\mathbb{Q}$, or the group of $p$-adic integers for any prime $p$, or $\mathbb{Z}^{\omega}$.

In the statement of the above proposition, note that condition (22) is formally weaker than (23) in two ways: it allows an arbitrary index set $I$, and it excludes factorization only through countably complete ultraproducts (which in the context $I=\omega$ of (23) would mean principal ultraproducts, i.e., the given groups $A_{n}$ ). Since (22) and (23) are equivalent, they are also equivalent to two intermediate conditions: the one obtained from (23) by replacing "ultraproducts (principal or 
nonprincipal)" by "principal ultraproducts", and the one obtained from (22) by deleting the words "countably complete".

We can deduce from these observations that the two sorts of conditions on an abelian group $B$ that we are interested in - namely, (a) that maps to $B$ from infinite direct products factor through finitely many ultraproducts, and (b) that in the case of a countable product, if we have a such a factorization, the ultrafilters involved are all principal - are not independent; precisely, that (a) implies (b). Indeed, (a) is equivalent to the negation of the version of (22) without the "countably complete" condition, which by the above observations is equivalent to the negation of the version of (23) in which the ultrafilters are assumed principal, i.e., the statement that every homomorphism from a countable product into $B$ factors through finitely many $A_{n}$, which clearly entails (b). Bringing in (24), we see that both (a) and (a) $\wedge$ (b) are equivalent to slenderness.

On the other hand, the three cases not excluded by the implication (a) $\Longrightarrow$ (b) all do occur. Slender groups, such as $\mathbb{Z}$, satisfy both (a) and (b). An infinite direct product of nontrivial slender groups, e.g., $\mathbb{Z}^{\omega}$, satisfies (b) but not (a). Finally, any nonprincipal countable ultraproduct of nontrivial abelian groups will not satisfy (b), hence, since (a) $\Longrightarrow$ (b), it will satisfy neither.

Having characterized the abelian groups $B$ that satisfy (a), it remains to characterize the larger class satisfying (b). As preparation, we shall first study the abelian groups that are homomorphic images of a single nonprincipal countable ultraproduct. We will need a few more definitions from the theory of infinite abelian groups.

Definition 22 [Fuchs 1970; Rotman 2009]. A subgroup B of an abelian group $A$ is called pure if for every positive integer $n, B \cap n A=n B$.

An abelian group $B$ is said to be algebraically compact if for every overgroup $A \supseteq B$ in which $B$ is pure, $B$ is a direct summand in A; equivalently [Fuchs 1970, Theorem 38.1], if for every set $X$ of group equations in constants from $B$ and $B$-valued variables, such that every finite subset of $X$ has a solution in $B$, the whole set $X$ has a solution in $B$.

An abelian group $B$ is said to be cotorsion if for every overgroup $A \supseteq B$ such that $A / B$ is torsion-free (a stronger condition than $B$ being pure in $A$ ), $B$ is a direct summand in $A$.

Of the two definitions of algebraic compactness quoted above, the first is the one commonly used. I include the second because it motivates the name of the condition. The theorem cited for their equivalence establishes several other diverse conditions as also equivalent to algebraic compactness; below, I shall pull these out of a hat as needed. 
The cotorsion abelian groups clearly include the algebraically compact abelian groups. In fact, they are precisely the homomorphic images of such groups [Fuchs 1970, Proposition 54.1], a fact called on in condition (30) in the next result.

Proposition 23. For B an abelian group, the following conditions are equivalent.

(25) There exists a family of abelian groups $\left(A_{i}\right)_{i \in I}$ and a non-countably-complete ultrafilter $U$ on I such that $B$ is a homomorphic image of the ultraproduct $\prod_{i \in I} A_{i} / \boldsymbol{u}$.

(26) There exists a countable family of abelian groups $\left(A_{n}\right)_{n \in \omega}$ and a nonprincipal ultrafilter $U$ on $\omega$ such that $B$ is a homomorphic image of the ultraproduct $\prod_{n \in \omega} A_{n} / \mathcal{u}$.

(27) There exists a countable family of abelian groups $\left(A_{n}\right)_{n \in \omega}$ and a filter F F on $\omega$ which is not contained in any principal ultrafilter (i.e., which satisfies $\left.\bigcap_{S \in \mathscr{F}} S=\varnothing\right)$, such that $B$ is a homomorphic image of $\prod_{n \in \omega} A_{n} / \mathscr{F}_{\text {. }}$

(28) There exists a countable family of abelian groups $\left(A_{n}\right)_{n \in \omega}$ such that $B$ is a homomorphic image of the reduced product $\left(\prod_{n \in \omega} A_{n}\right) / \bigoplus_{n \in \omega} A_{n}$.

(29) $B$ is a homomorphic image of an abelian group $C$ admitting a compact Hausdorff group topology.

(30) B is a cotorsion abelian group; i.e., a homomorphic image of an algebraically compact abelian group.

Proof. We shall show (25) $\Longrightarrow$ (26) $\Longrightarrow$ (27) $\Longrightarrow$ (28) $\Longrightarrow$ (30) $\Longrightarrow$ (29) $\Longrightarrow$ (25).

In the situation of (25), the fact that $U$ is not countably complete implies that we can find a partition $I=\bigcup_{n \in \omega} J_{n}$ such that no $J_{n}$ belongs to $U$. Let us again write $\prod_{i \in I} A_{i}=\prod_{n \in \omega}\left(\prod_{i \in J_{n}} A_{i}\right)$. As in the proof of Lemma 10, if we let $u^{\prime}=$ $\left\{S \subseteq \omega \mid \bigcup_{n \in S} J_{n} \in U\right\}$, we find that $\mathcal{U}^{\prime}$ is a nonprincipal ultrafilter on $\omega$, yielding a factorization of the map from our product group to our original ultraproduct as

$$
\prod_{i \in I} A_{i} \rightarrow \prod_{n \in \omega}\left(\prod_{i \in J_{n}} A_{i}\right) / \mathcal{U}^{\prime} \rightarrow \prod_{i \in I} A_{i} / \mathcal{U} .
$$

Since $B$ is a homomorphic image of $\prod_{i \in I} A_{i} / \mathcal{U}$, it is a homomorphic image of the factoring object, proving (26).

We get $(26) \Longrightarrow(27)$ by taking $\mathscr{F}=U$.

Given (27), note that since the filter $\mathscr{F}$ on $\omega$ is not contained in a principal ultrafilter, it contains the complement of every singleton, hence it contains the Fréchet filter $\mathscr{C}$ of complements of finite sets. So the quotient map

$$
\prod_{n \in \omega} A_{n} \rightarrow \prod_{n \in \omega} A_{n} / \mathscr{F} \quad \text { factors through } \prod_{n \in \omega} A_{n} / \mathscr{C}=\left(\prod_{n \in \omega} A_{n}\right) / \bigoplus_{n \in \omega} A_{n},
$$

giving (28). 
Given (28), we call on [Fuchs 1970, Corollary 42.2] which says that every group of the form $\left(\prod_{n \in \omega} A_{n}\right) / \bigoplus_{n \in \omega} A_{n}$ is algebraically compact, yielding (30).

For the step (30) $\Longrightarrow$ (29), we call on [Fuchs 1970, Theorem 38.1] (or on [Rotman 2009, Theorem 7.42]) which, among the equivalent conditions for an abelian group to be algebraically compact, includes that of being a direct summand in an abelian group that admits a compact Hausdorff group topology. So an algebraically compact abelian group is, in particular, a homomorphic image of an abelian group admitting such a topology, hence so is any homomorphic image of an algebraically compact group.

Finally, by Proposition 15 above, any abelian group $A$ admitting a compact Hausdorff group topology can be written as a homomorphic image of its ultrapower $A^{I} / \mathcal{U}$ for any ultrafilter $U$ on any set $I$. So choosing a $U$ which is not countably complete (e.g., any nonprincipal ultrafilter on $I=\omega$ ), we get (29) $\Longrightarrow(25)$.

We note that for a nonzero abelian group $B$, the equivalent conditions of Proposition 23 imply those of Proposition 21. Indeed, thinking in terms of the conditions (a) and (b) that we have been discussing, if we write $\left(b_{1}\right)$ for the case of (b) where there is only a single ultraproduct involved (i.e., the condition that if there exists a nonzero homomorphism from an ultraproduct group $\prod_{n \in \omega} A_{n} / \mathcal{U}$ onto $B$, then the ultrafilter $U$ is principal), then we have $(a) \Longrightarrow(b) \Longrightarrow\left(b_{1}\right)$, so $\neg\left(\mathrm{b}_{1}\right) \Longrightarrow \neg(\mathrm{a})$; moreover, we see that for $B \neq\{0\},(26)$ is equivalent to $\neg\left(\mathrm{b}_{1}\right)$, while we have previously noted that the conditions of Proposition 21 are equivalent to $\neg$ (a). (Alternatively, it not hard to see directly that for $B \neq\{0\}$, an example witnessing (26) also witnesses (22).) Choosing the equivalent conditions of the two propositions that have standard names, these observations say that for $B \neq\{0\},(30) \Longrightarrow$ (24); in other words, no nonzero cotorsion abelian group is slender. Since the class of cotorsion abelian groups is closed under homomorphic images, this in fact gives:

Corollary 24. No cotorsion abelian group has a nonzero slender homomorphic image.

Corollary 24 allows us to apply Theorem 13 to many variants of the examples immediately following it. For instance, one of those was the infinite dihedral group, i.e., the semidirect product arising from the natural action of $\{ \pm 1\}$ on the slender group $\mathbb{Z}$. I claim that we can replace $\mathbb{Z}$ in that example by any abelian group $A$ without 2-torsion that has $\mathbb{Z}$ as a homomorphic image; for instance, $\mathbb{Z}^{\omega}$, or $\mathbb{Z} \times \mathbb{Z} / n \mathbb{Z}$ for any odd $n$. Indeed, taking a homomorphism from such an abelian group $A$ onto $\mathbb{Z}$, and any $b \in A$ that maps to a generator of $\mathbb{Z}$ under that homomorphism, we see from the above corollary that no subgroup $B$ of $A$ containing $b$ is cotorsion, equivalently, by Proposition 23, that no such subgroup $B$ satisfies (26); hence $b$ satisfies the condition of the second paragraph of Theorem 13. (The assumption that $A$ has no 2-torsion keeps the center of the semidirect product trivial, to avoid 
complicating our considerations.) In Section 6 we will obtain more information on which abelian groups are cotorsion.

We can now answer the question of which abelian groups $B$ have the property we called (b) in our earlier discussion, namely, that any map $f$ from a countable direct product of abelian groups $A_{n}$ onto $B$ which factors through finitely many ultrafilters in fact factors through the projection to the product of finitely many of the $A_{n}$. We shall see that this is true if and only if $B$ contains no nontrivial cotorsion subgroup. Although the class of cotorsion abelian groups is difficult to describe exactly, a simple criterion is known for an abelian group to be cotorsion-free, i.e., to contain no nontrivial cotorsion subgroup: It is that the group be torsion-free, and contain no copy of the additive group of $\mathbb{Q}$, nor of the $p$-adic integers for any prime $p$ [Dugas and Göbel 1982, Theorem $2.4(1) \Longrightarrow(4)$ ]. (So it is like the condition characterizing slenderness, but without the exclusion of subgroups isomorphic to $\mathbb{Z}^{\omega}$.) This condition is also equivalent to that of containing no nonzero algebraically compact subgroup: it implies the latter because every algebraically compact group is cotorsion, while the reverse implication holds because $\mathbb{Q}$, and the groups of $p$-adic integers, and all finite abelian groups, are algebraically compact. As is usual in this note, the statement below will be the contrapositive of the version suggested by this discussion.

Theorem 25. The following conditions on an abelian group $B$ are equivalent.

(31) There exist a set $I$, a family of abelian groups $\left(A_{i}\right)_{i \in I}$, and a surjective homomorphism $f: \prod_{i \in I} A_{i} \rightarrow B$ such that $f$ factors through the product of finitely many ultraproducts $\prod_{i \in I} A_{i} / \cup_{k}$, but does not factor through the product of finitely many countably complete ultraproducts.

(32) There exist a countable family $\left(A_{n}\right)_{n \in \omega}$ of abelian groups and a surjective homomorphism $f: \prod_{n \in \omega} A_{n} \rightarrow B$ such that $f$ factors through the product of finitely many ultraproducts $\prod_{n \in \omega} A_{n} / \mathcal{U}_{k}$, but does not factor through the product of finitely many of the $A_{n}$.

(33) $B$ has a nontrivial cotorsion subgroup; equivalently (by the result from [Dugas and Göbel 1982] quoted above), B either has nonzero elements of finite order, or contains a copy of the additive group of $\mathbb{Q}$, or contains a copy of the additive group of the p-adic integers for some prime $p$; equivalently, $B$ has a nontrivial algebraically compact subgroup.

These conditions are also equivalent to the variants of (32) and (31) without the assumption that $f$ be surjective.

Proof. The equivalence of (31) and (32) to the corresponding conditions without the assumption of surjectivity is seen as in the first paragraph of the proof of Proposition 21 . We shall use those variants to prove (33) $\Longrightarrow(32) \Longrightarrow(31) \Longrightarrow(33)$. 
Assuming (33), let $C \subseteq B$ be a nonzero cotorsion subgroup. Then our earlier result (30) $\Longrightarrow$ (26) gives a surjective homomorphism $\prod_{n \in \omega} A_{n} / \mathcal{U} \rightarrow C$ for a family of abelian groups $A_{n}$ and a nonprincipal ultrafilter $U$, which we regard as a nonzero homomorphism into $B$. Since $U$ is not principal, $f$ annihilates each of the $A_{n}$, so it cannot be factored through the product of finitely many of these, giving (32).

Clearly, (32) $\Longrightarrow(31)$, since the countably complete ultrafilters on $\omega$ are the principal ultrafilters.

Assuming (31), let $f: \prod_{i \in I} A_{i} \rightarrow B$ be a homomorphism that factors through a product of ultraproducts $\prod_{i \in I} A_{i} / \mathcal{u}_{0} \times \cdots \times \prod_{i \in I} A_{i} / u_{m-1}$, but not through such a product in which all the $U_{k}$ are countably complete. As noted in the proof of Proposition 21, the given factorization is equivalent to an expression of $f$ as the sum of maps that factor $\prod_{i \in I} A_{i} \rightarrow \prod_{i \in I} A_{i} / u_{k} \rightarrow B$, and if any of these maps are zero, we can drop them, leaving a factorization with all these maps nonzero, and which, by choice of $f$, must still have at least one with $\varkappa_{k}$ not countably complete. So there exists a nonzero map $g: \prod_{i \in I} A_{i} / \mathcal{U} \rightarrow B$ for some non-countably-complete ultrafilter $U$ on $I$. Our earlier result (25) $\Longrightarrow$ (30) now tells us that the nonzero image of $g$ is a cotorsion submodule of $B$, proving (33).

We have not yet said much about algebraically compact groups, except that the cotorsion groups are their homomorphic images. We record:

Lemma 26. The following conditions on an abelian group B are equivalent.

(34) For every proper filter $\mathscr{F}$ on a nonempty set $I$, the natural embedding $B \rightarrow$ $B^{I} / \mathscr{F}$ has a left inverse.

(35) There exists a nonprincipal ultrafilter $\mathcal{U}$ on $\omega$ such that the natural embedding $B \rightarrow B^{\omega} / \mathcal{U}$ has a left inverse.

(36) B is algebraically compact.

Proof. For any filter $\mathscr{F}$ on a set $I$, the natural embedding $B \rightarrow B^{I} / \mathscr{F}$ is easily seen to be pure, so the definition of algebraic compactness gives (36) $\Longrightarrow(34)$. Clearly, (34) $\Longrightarrow$ (35).

To show that (35) $\Longrightarrow$ (36), we use the result [Eklof 1973, third sentence of §2], that a nonprincipal countable ultrapower of any abelian group $B$ is algebraically compact. Hence (35) implies that $B$ is a direct summand in an algebraically compact abelian group, from which one easily sees that it itself is algebraically compact.

Since the cotorsion abelian groups are the homomorphic images of the algebraically compact ones, the above result shows that the analog of Question 16 has a positive answer for abelian groups. (This can also be seen from the proof of Proposition 23, where the closing step (29) $\Rightarrow(25)$ allows us to choose $u$ essentially arbitrarily.) 
Another interesting necessary and sufficient condition for $B$ to be algebraically compact, obtained (in the more general context of modules) as [Jensen and Lenzing 1989, Theorem 7.1(vi)], is that for every set $I$, the summation map $\bigoplus_{i \in I} B \rightarrow B$ extend to a map $B^{I} \rightarrow B$.

\section{More on algebraically compact and cotorsion abelian groups}

The distinction between the class of cotorsion abelian groups and its subclass, the algebraically compact abelian groups, is a subtle one. It follows from the definitions that every cotorsion abelian group $B$ that is torsion-free is algebraically compact [Fuchs 1970, Corollary 54.5]. The only example I have found in the literature of a cotorsion abelian group that is not algebraically compact, that of [Rotman 2009, Proposition 7.48(ii)], is described as an Ext of other groups, rather than explicitly. (It is known that for any abelian groups $A$ and $A^{\prime}, \operatorname{Ext}\left(A, A^{\prime}\right)$ is cotorsion [Fuchs 1970, Theorem 54.6], [Rotman 2009, Corollary 7.47].) Let us begin this section by constructing a more explicit example.

We will use the characterization of an algebraically compact abelian group as an abelian group $B$ such that whenever a system of equations has the property that all its finite subsystems have solutions in $B$, then the whole system has such a solution. An easy example of an infinite system of equations is the following, where $p$ is a prime, $x_{0}$ is a given element of $B$, and $x_{1}, \ldots, x_{n}, \ldots$ are to be found.

$$
x_{0}=p x_{1}, \quad x_{1}=p x_{2}, \quad \ldots, \quad x_{n-1}=p x_{n}, \quad \ldots
$$

The necessary condition for algebraic compactness that this system yields is:

Lemma 27. If $B$ is an algebraically compact group and $p$ a prime, then the subgroup $B^{\prime}=\bigcap_{n \in \omega} p^{n} B \subseteq B$ is p-divisible, i.e., satisfies $p B^{\prime}=B^{\prime}$.

Proof. Suppose $x_{0} \in B^{\prime}$. Let us fix $n \geq 0$, and choose $x_{n} \in B$ such that $x_{0}=p^{n} x_{n}$. If we now let $x_{m}=p^{n-m} x_{n}$ for $0<m<n$, we see that $x_{0}, \ldots, x_{n}$ satisfy the first $n$ equations of (37). Since we can do this for any $n$, every finite subfamily of (37) has a solution, so algebraic compactness implies that we can choose $x_{1}, \ldots, x_{n}, \ldots$ satisfying the full set of equations. For such $x_{1}, \ldots, x_{n}, \ldots$ we see that $x_{1}$ also belongs to $B^{\prime}$; so $x_{0} \in p B^{\prime}$, as required.

(It is also not hard to prove the above lemma from the definition of algebraic compactness in terms of pure extensions: given algebraically compact $B$, and $x_{0} \in B^{\prime}$, let $B^{+}$be the extension of $B$ gotten by adjoining new generators $x_{1}, \ldots, x_{n}, \ldots$ and the relations (37). It is straightforward to show that $B$ embeds in $B^{+}$, and from the fact that $x_{0} \in B^{\prime}$, one can deduce that $B$ is pure in $B^{+}$. Hence the definition of algebraic compactness says that there exists a retraction of $B^{+}$onto $B$, i.e., a solution to (37) in $B$; hence, as above, $x_{0}=p x_{1} \in p B^{\prime}$.) 
So let us try to construct a cotorsion abelian group $B$ with an element that we force to lie in $B^{\prime}$, without creating any apparent reason why it should lie in $p B^{\prime}$. To do this, let $\mathbb{Z}_{p}$ denote the additive group of $p$-adic integers, which is algebraically compact by Proposition 15 and Lemma 26; within its countable power $\mathbb{Z}_{p}^{\omega}$, let $\delta_{n}$ be, as usual, the element with 1 in the $n$-th coordinate and 0 in all others; and for a first try, let $B$ be the factor group of $\mathbb{Z}_{p}^{\omega}$ by the subgroup generated by the elements

$$
\delta_{0}-p^{n} \delta_{n} \quad(n \in \omega) .
$$

Letting $x$ be the image of $\delta_{0}$ in $B$, we clearly have $x \in B^{\prime}$.

But this group is messy, making it hard to see whether some $y \in B^{\prime}$ might satisfy $x=p y$. It becomes nicer if we impose (38) as $\mathbb{Z}_{p}$-module relations rather than just as additive group relations. If we then change coordinates in $\mathbb{Z}_{p}^{\omega}$, so that the elements $\delta_{n}-p \delta_{n+1}$ become the new $\delta_{n}$ (namely, we map $\left(a_{n}\right)_{n \in \omega}$ to $\left.\left(\sum_{m \leq n} p^{n-m} a_{m}\right)_{n \in \omega}\right)$, the resulting construction takes the form shown in the next lemma.

Lemma 28. Let $p$ be a prime number, and $B$ the group $\mathbb{Z}_{p}^{\omega} / \bigoplus_{n \in \omega} p^{n} \mathbb{Z}_{p}$. Then $B$ is cotorsion, but fails to satisfy the conclusion of Lemma 27; hence $B$ is not algebraically compact.

Proof. As a homomorphic image of an algebraically compact group, $B$ is cotorsion.

To see the failure of the conclusion of Lemma 27, let $x \in B$ be the image of $\left(p^{n}\right)_{n \in \omega} \in \mathbb{Z}_{p}^{\omega}$. (Note that the above coordinates $p^{n}$ are "ghosts", in the sense that any finite set of them may, by the definition of $B$, be changed to 0 without changing the element $x$.) For each $n>0$, if we let $x_{n} \in B$ be the image of the element of $\mathbb{Z}_{p}^{\omega}$ whose coordinate in position $m$ is 0 for $m<n$, and $p^{m-n}$ for $m \geq n$, then we see that $x=p^{n} x_{n}$. Hence $x \in B^{\prime}$.

Now let $y$ be any element satisfying $x=p y$. Writing $y$ as the image of $\left(a_{n}\right)_{n \in \omega} \in \mathbb{Z}_{p}^{\omega}$, we see from the definition of $B$ that for all but finitely many $n$ we must have $a_{n}=p^{n-1}$. (And note that coordinates with this property are not "ghosts"!) But for any $n$ such that this equation holds, we can see by looking at the $n$-th coordinate that $y \notin p^{n} B$. So $y \notin B^{\prime}$; and since we have shown this for all $y$ with $x=p y$, we have $x \notin p B^{\prime}$. Since $x \in B^{\prime}$, this shows that $B^{\prime} \neq p B^{\prime}$.

(L. Fuchs (personal communication) points out another way to see that the above group $B$ is not algebraically compact: by noting that its torsion subgroup $\bigoplus_{n \in \omega} \mathbb{Z}_{p} / p^{n} \mathbb{Z}_{p}$ is not torsion-complete, and calling on [Fuchs 1973, Theorem 68.4, (ii) $\Longrightarrow$ (i)].)

Note that any group $B$ which, like the one constructed above, is cotorsion but not algebraically compact is, by the former fact, a homomorphic image of a nonprincipal countable ultraproduct of groups, but by Lemma 26 (35) $\Longrightarrow$ (36), does not admit a left inverse to a diagonal embedding $B \rightarrow B^{\omega} / \mathcal{U}$, confirming the assertion made in the second paragraph after Question 17. 
Let us obtain, next, some restrictions on the class of cotorsion abelian groups. These will allow us to deduce that many sorts of groups are not cotorsion, and so give more examples to which we can apply Theorem 13. In the next lemma we combine the fact that the cotorsion groups are the homomorphic images of the algebraically compact groups with another of the criteria for algebraic compactness given in [Fuchs 1970, Theorem 38.1], namely, that an abelian group $C$ is algebraically compact if and only if it is pure-injective, meaning that for any pure subgroup $A_{0}$ of an abelian group $A_{1}$, every homomorphism $A_{0} \rightarrow C$ extends to a homomorphism $A_{1} \rightarrow C$. In an earlier version of this note, I asked whether the direction "(39) $\Longrightarrow$ cotorsion" in the lemma held; I am indebted to K. M. Rangaswamy and Manfred Dugas for (independently) showing me why it does.

Lemma 29. An abelian group $B$ is cotorsion if and only if it satisfies

(39) For every abelian group A having a pure subgroup $F$ which is free abelian, every homomorphism $F \rightarrow B$ extends to a homomorphism $A \rightarrow B$.

Proof. Assuming $B$ cotorsion, let us write it as a homomorphic image of an algebraically compact abelian group $C$. Since $F$ is free, we can lift the given map $F \rightarrow B$ to a map $F \rightarrow C$, and then, since $C$ is algebraically compact, equivalently, pure-injective, we can extend that lifted map to a map $A \rightarrow C$. Composing with our map $C \rightarrow B$, we get the desired extension to $A$ of the given map $F \rightarrow B$.

Conversely, assuming (39), write $B$ as a homomorphic image of a free abelian group $F$. Now by [Fuchs 1970, §38, Exercise 8, p. 162], every abelian group embeds as a pure subgroup in a group admitting a compact Hausdorff group topology; let $A$ be such an overgroup of $F$. (For an explicit embedding in this case, let $\widehat{\mathbb{Z}}$ denote the completion of $\mathbb{Z}$ with respect to its subgroup topology. Then $\mathbb{Z}$ is a pure subgroup of the compact group $\widehat{\mathbb{Z}}$, so writing $F=\bigoplus_{I} \mathbb{Z}$, we see that $F$ is pure in the compact group $\widehat{\mathbb{Z}}^{I}$.) By (39), our homomorphism of $F$ onto $B$ extends to a homomorphism of $A$ onto $B$, so by Proposition 23, (29) $\Longrightarrow(30), B$ is cotorsion.

Our first application of this result will show that in a cotorsion abelian group $B$, highly divisible elements abound; for instance, that if $p_{1}$ and $p_{2}$ are distinct primes, then every element of $B$ is the sum of an element divisible by all powers of $p_{1}$ and an element divisible by all powers of $p_{2}$. To state the result in greater generality, let us, for any set $P$ of primes, write $\mathbb{Z}\left[P^{-1}\right]$ for the subring of $\mathbb{Q}$ consisting of elements whose denominators lie in the multiplicative monoid generated by $P$, and call an element $x$ of an abelian group $A P$-divisible if it lies in the image of a homomorphism from the additive group of $\mathbb{Z}\left[P^{-1}\right]$ to $A$. We shall call an abelian group $P$-divisible if all its elements are.

Proposition 30. If $B$ is a cotorsion abelian group, and $P_{0}, \ldots, P_{m-1}$ are sets of prime numbers such that $P_{0} \cap \cdots \cap P_{m-1}=\varnothing$, then every element $b \in B$ can be 
written $b_{0}+\cdots+b_{m-1}$, where for each $j, b_{j}$ is $P_{j}$-divisible. Equivalently, $B$ is a sum of subgroups $B_{0}+\cdots+B_{m-1}$ such that each group $B_{j}$ is $P_{j}$-divisible.

Proof. Let $A$ be the additive group of $\mathbb{Z}\left[P_{0}^{-1}\right] \times \cdots \times \mathbb{Z}\left[P_{m-1}^{-1}\right]$, and $F$ the infinite cyclic subgroup thereof generated by $(1, \ldots, 1)$. That the inclusion $F \subseteq A$ is pure follows from the fact that $P_{0} \cap \cdots \cap P_{m-1}=\varnothing$. Indeed, if an element $d(1, \ldots, 1) \in F$ is not divisible in $F$ by some positive integer $n$, then $d$ is not divisible by $n$, so $n$ has a prime power factor $p^{i}$ not dividing $d$. Choosing $k$ such that $p \notin P_{k}$, we see that the $k$-th coordinate of $d(1, \ldots, 1)$ is not divisible by $p^{i}$ in $\mathbb{Z}\left[P_{k}^{-1}\right]$, so in $A$, $d(1, \ldots, 1)$ is not divisible by $p^{i}$, hence not divisible by $n$.

Hence by Lemma 29 , for any $b \in B$, the map $F \rightarrow B$ taking $(1, \ldots, 1)$ to $b$ extends to $A$, giving a representation of $b$ as the sum of the images of the elements $(0, \ldots, 1, \ldots, 0)$, each of which is $P_{j}$-divisible for some $j$. The equivalence of this result to the final statement of the lemma follows from the fact that for any set $P$ of primes, the $P$-divisible elements of an abelian group form a subgroup.

As a quick illustration, consider the group $\mathbb{Z}_{p}$ of $p$-adic integers, which we have seen is algebraically compact, and hence cotorsion. That group is $P$-divisible for $P$ the set of all primes other than $p$. Given $P_{0}, \ldots, P_{m-1}$ as in Proposition 30, at least one $P_{j}$ will fail to contain $p$, so $\mathbb{Z}_{p}$ is $P_{j}$-divisible for that $j$, confirming the conclusion of the proposition.

Of course, the much smaller group of rational numbers with denominators relatively prime to $p$ (of which the group $\mathbb{Z}_{p}$ is a completion) is $P$-divisible for the same set $P$, and so also satisfies the conclusion of Proposition 30. However, that group is not cotorsion. Indeed, from the characterization of slender abelian groups recalled immediately after the proof of Proposition 21, every abelian group which is torsion-free and which contains no copy of $\mathbb{Q}$ and has less than continuum cardinality is slender, hence, if nonzero, is non-cotorsion.

The next result generalizes the above restriction on cotorsion groups.

Proposition 31. If $B$ is a cotorsion abelian group such that $d B \neq\{0\}$ for every positive integer $d$, but $\bigcap_{d \in \mathbb{Z}, d>0} d B=\{0\}$, then $B$ has at least continuum cardinality. Proof. We shall construct a homomorphism $\bigoplus_{\omega} \mathbb{Z} \rightarrow B$, extend it to a map $\mathbb{Z}^{\omega} \rightarrow B$ by Lemma 29 , and show that under the extended map, continuum many elements of $\mathbb{Z}^{\omega}$ have distinct images. We begin by carefully selecting the elements to which to send the free generators of $\bigoplus_{\omega} \mathbb{Z}$.

I claim that we can choose positive integers $d_{0}, d_{1}, \ldots$, each a multiple of the one before, and elements $b_{0}, b_{1}, \ldots \in B$, such that for each $n \in \omega$, we have $d_{n} b_{n} \notin d_{n+1} B$. We start with $d_{0}=1$, and $b_{0}$ any nonzero element of $B$. Assuming that for some $n \geq 0, d_{n}$ and $b_{n}$ have been chosen with $d_{n} b_{n} \neq 0$, the hypothesis $\bigcap_{d \in \mathbb{Z}, d>0} d B=\{0\}$ allows us to choose $d_{n+1}>0$ such that $d_{n} b_{n} \notin d_{n+1} B$. Replacing $d_{n+1}$ by a proper multiple if necessary, we may assume $d_{n} \mid d_{n+1}$. Using the fact that $d_{n+1} B \neq\{0\}$, 
we can then choose $b_{n+1}$ such that $d_{n+1} b_{n+1} \neq 0$. Continuing recursively, we get $d_{0}, d_{1}, \ldots$ and $b_{0}, b_{1}, \ldots$ with the asserted properties.

We now map $\bigoplus_{\omega} \mathbb{Z}$ to $B$ by sending each $\delta_{n}$ to $b_{n}$. Since $\bigoplus_{\omega} \mathbb{Z}$ is a pure subgroup of $\mathbb{Z}^{\omega}$, Lemma 29 allows us to extend this map to a homomorphism $f: \mathbb{Z}^{\omega} \rightarrow B$, which still carries each $\delta_{n}$ to $b_{n}$.

For each $\varepsilon=\left(\varepsilon_{n}\right)_{n \in \omega} \in\{0,1\}^{\omega}$, let $\varepsilon d$ denote $\left(\varepsilon_{0} d_{0}, \ldots, \varepsilon_{n} d_{n}, \ldots\right) \in \mathbb{Z}^{\omega}$. I claim that distinct strings $\varepsilon$ yield distinct elements $f(\varepsilon d) \in B$. Indeed, for $\varepsilon \neq \varepsilon^{\prime}$, let $n \in \omega$ be the least index such that $\varepsilon_{n} \neq \varepsilon_{n}^{\prime}$, and let us write

$$
f(\varepsilon d)=f\left(\varepsilon_{0} d_{0}, \ldots, \varepsilon_{n} d_{n}, 0,0, \ldots\right)+f\left(0, \ldots, 0, \varepsilon_{n+1} d_{n+1}, \varepsilon_{n+2} d_{n+2}, \ldots\right) .
$$

If we compare this with the corresponding expression for $f\left(\varepsilon^{\prime} d\right)$, we see that the left-hand summands in these expressions differ by exactly $f\left(d_{n} \delta_{n}\right)$, i.e., $d_{n} b_{n}$, which by assumption does not lie in $d_{n+1} B$; while the right-hand summands $d o$ lie in $d_{n+1} B$, since for all $m \geq n$ we have $d_{n+1} \mid d_{m}$. Hence $f(\varepsilon d)-f\left(\varepsilon^{\prime} d\right) \neq 0$; so we indeed have continuum many distinct elements of $B$.

As an application, it is easy to deduce that no subgroup $B$ of $\prod_{\text {primes } p} \mathbb{Z} / p \mathbb{Z}$ which is infinite, but of less than continuum cardinality, can be cotorsion. Hence, if we take such a subgroup with no 2-torsion, containing an element $b$ of infinite order, its semidirect product with \pm 1 will again be a group to which Theorem 13 applies.

On the other hand, we saw in Lemma 11 that for the semidirect product of $\{ \pm 1\}$ with the group $\mathbb{Q}$, the conclusion of Theorem 13 fails; and Proposition 15 shows the same for the semidirect product of $\{ \pm 1\}$ with any finite abelian group. In fact, $\mathbb{Q}$ and all finite abelian groups are cotorsion; the next result includes these statements as special cases. It is curious that its formulation is analogous to that of Proposition 15, but the reasoning is quite different.

Proposition 32 (cf. [Fuchs 1970, p. 178, last paragraph of Notes]). Let B be an abelian group which is divisible, or is of finite exponent, or more generally, is the sum of a divisible group and one of finite exponent; or, still more generally, is the underlying additive group of an injective module over some ring $R$. Then for any set $I$ and any ultrafilter $U$ on $I$, there is a group homomorphism $B^{I} / U \rightarrow B$ left-inverse to the natural embedding $B \rightarrow B^{I} \rightarrow B^{I} /$ U.

Hence by Lemma $26,(35) \Longrightarrow(36)$, every such $B$ is algebraically compact, and so in particular is cotorsion.

Proof. First suppose $B$ has the property introduced above by the words "still more generally". Then the maps $B \rightarrow B^{I} \rightarrow B^{I} / \mathcal{U}$ are $R$-module homomorphisms whose composite is an embedding. The injectivity of $B$ as an $R$-module thus yields the desired left inverse map. Taking $I=\omega$ and $U$ nonprincipal, we conclude that $B$ is algebraically compact (by Lemma $26,(35) \Longrightarrow(36)$ ). 
It remains to show that the various sorts of abelian groups named are indeed injective modules over appropriate rings. Any divisible abelian group is an injective $\mathbb{Z}$-module by [Lam 1999, Proposition 3.19]. An abelian group $B$ of finite exponent $n$ can be written as a direct product of free $\mathbb{Z} / d \mathbb{Z}$-modules as $d$ ranges over the divisors of $n$; and each of the rings $\mathbb{Z} / d \mathbb{Z}$ is self-injective, so that its free modules are injective by [ibid., Corollary 3.13(1) and Theorem 3.46(4) $\Longrightarrow$ (2)]. Finally, if $B$ is the sum of a divisible subgroup $D$ and a subgroup $E$ of finite exponent, then the injectivity of $D$ over $\mathbb{Z}$ allows us to split it off as a direct summand, and the complementary summand will be a homomorphic image $E^{\prime}$ of $E$, hence again of finite exponent. We can now make $B=D \oplus E^{\prime}$ a module over the direct product $R$ of $\mathbb{Z}$ and finitely many rings $\mathbb{Z} / d \mathbb{Z}$, in such a way that the component over each of these factor rings is injective over that ring. The group $B$ will then be injective over $R$.

I do not know the answer to: ${ }^{1}$

Question 33. For an abelian group $B$ to be cotorsion, is it sufficient that every homomorphism $\bigoplus_{\omega} \mathbb{Z} \rightarrow B$ extend to a homomorphism $\mathbb{Z}^{\omega} \rightarrow B$ ? (In other words, in Lemma 29, is condition (39) equivalent to the special case where the inclusion $F \subseteq A$ is $\bigoplus_{\omega} \mathbb{Z} \subseteq \mathbb{Z}^{\omega}$ ?)

The following example shows that the converse of Corollary 24 is not true: a group $B$ with no nonzero slender homomorphic image need not be cotorsion.

Lemma 34. Within the group $A=\prod_{\text {primes } p} \mathbb{Z} / p \mathbb{Z}$, let $u$ be the element having 1 in every coordinate, and let $B$ consist of all elements $b \in A$ such that $d b=n u$ for some integer $n$ and nonzero integer $d$ (mnemonic for "numerator" and "denominator").

Then $B$ is a countable subgroup of $A$, such that every cotorsion subgroup of $B$ is torsion (so that $B$ is not itself cotorsion), but the factor-group of $B$ by its torsion subgroup is isomorphic to $\mathbb{Q}$, and so is cotorsion.

Hence $B$ has no nonzero slender homomorphic images.

Proof. That $B$ is a subgroup of $A$ is immediate. It is countable because each $b \in B$ is determined by any choice of $n$ and $d$ satisfying $d b=n u$, together with the coordinates of $b$ at the finitely many primes dividing $d$.

By the observation following the proof of Proposition 31, cotorsion subgroups of $B$ are finite, hence are torsion.

On the other hand, the factor group of $B$ by its torsion subgroup is isomorphic to $\mathbb{Q}$ via the map sending the image of each $b \in B$ to the common value of $n / d \in \mathbb{Q}$ for all relations $d b=n u$ satisfied by $b$; and $\mathbb{Q}$, being divisible, is cotorsion by Proposition 32.

${ }^{1}$ See note added in proof, page 494. 
Since slender groups are torsion-free, a homomorphism $f$ from $B$ to a slender group must annihilate the torsion subgroup of $B$, hence $f(B)$ must be a homomorphic image of $\mathbb{Q}$, hence by Corollary 24 must be zero.

Here is a question of a different flavor.

Question 35. If an abelian group B can be written as a homomorphic image of a nonprincipal countable ultraproduct of not necessarily abelian groups $G_{n}$, must it be a homomorphic image of a nonprincipal countable ultraproduct of abelian groups, i.e., must it be cotorsion?

The reason this question is nontrivial is that abelianization does not commute with ultraproducts. For instance, let $G$ be a group which is perfect (satisfies $G=[G, G]$ ) but which for each $n$ has an element $x_{n}$ that cannot be written as the product of fewer than $n$ commutators. (The latter property is called "infinite commutator width"; for examples of such $G$ see [Muranov 2007].) Then no nonprincipal ultrapower $G^{\omega} / \mathcal{U}$ will be perfect, because for such a family of elements $x_{n}$, the image of $\left(x_{n}\right)_{n \in \omega} \in G^{\omega}$ in $G^{\omega} / \mathcal{U}$ will not be a product of finitely many commutators. Hence the abelianization $B$ of $\prod_{n \in \omega} G_{n} / \mathcal{U}$ is a nontrivial abelian group satisfying the hypothesis of Question 35, but there is no obvious candidate for a representation of $B$ as in the conclusion of that question.

We can, however, prove a weak result in the direction of a positive answer.

Lemma 36. If an abelian group $B$ can be written as a homomorphic image of a nonprincipal countable ultraproduct $\prod_{n \in \omega} G_{n} / \mathcal{U}$ of not necessarily abelian groups, then $B$ is a directed union of cotorsion abelian subgroups.

Proof. By Lemma 12, every cyclic subgroup of $B$ is contained in a cotorsion subgroup. Now the class of cotorsion abelian groups, as characterized by any of (27), (28) or (29), is easily seen to be closed under finite direct sums, hence since it is closed under homomorphic images, it is closed under finite sums in abelian overgroups, so the cotorsion subgroups of such an overgroup form a directed system.

But not every directed union of cotorsion groups is cotorsion. For instance, every torsion abelian group is the directed union of its finite subgroups, which are cotorsion by Proposition 32 ; but by Proposition 31 , the group $\bigoplus_{\text {primes } p} \mathbb{Z} / p \mathbb{Z}$ is not cotorsion.

We remark that Questions 19 and 35 cannot both have positive answers, since as noted earlier, a positive answer to Question 19 would make every torsion group, including the abovementioned group $\bigoplus_{\text {primes } p} \mathbb{Z} / p \mathbb{Z}$, a homomorphic image of a countable ultraproduct of (not necessarily abelian) groups. But as we also said earlier, a positive answer to Question 19 seems highly unlikely.

A noticeable difference between our results on general groups in Sections 2-4 and our results on abelian groups in the above three sections is that in the former we 
composed maps $\prod_{i \in I} G_{i} \rightarrow B$ with the natural map $B \rightarrow B / Z(B)$ before looking at factorization properties, but we have done nothing of the sort for abelian groups. It might be of interest to see whether one can improve the results of these sections by composing homomorphisms $\prod_{i \in I} A_{i} \rightarrow B$ with the map $B \rightarrow B / X(B)$ for some natural choice of $X(B)$, such as the torsion subgroup of $B$, the subgroup of divisible elements, their sum, or the sum of all cotorsion subgroups of $B$. (Lemma 34 shows that for the last of these choices, $B / X(B)$ may not itself be cotorsion-free; but this need not be a problem; cf. the fact that for a nonabelian group $B$, the group $B / Z(B)$ need not have trivial center.) In the opposite direction, it might be possible to strengthen the results of Sections $2-4$ by dividing $B$, not by $Z(B)$, but by a smaller subgroup $X(Z(B))$ for one of the above constructions $X$. I leave these ideas for others to explore.

\section{Some related questions that have been studied}

The direct sum $\bigoplus_{i \in I} A_{i}$ of a family of abelian groups — or more generally, of a family of modules over any ring $R$ - is their coproduct in the category of abelian groups or $R$-modules; hence for such objects, their coproduct can be regarded as the subgroup or submodule of elements of finite support in their direct product $\prod_{i \in I} A_{i}$. Now in any category, a homomorphism from a coproduct of objects $A_{i}$ to an arbitrary object $B$ is determined simply by choosing a homomorphism from each $A_{i}$ to $B$. So the phenomena we have been investigating in the last two sections can be looked at as consequences of the fact that not every such map on a coproduct of abelian groups can be extended consistently to the elements of $\prod_{i \in I} A_{i}$ with infinite supports. The slender modules are those modules $B$ for which this restriction on maps to $B$ is so strong that it can only be satisfied by maps that factor through the product of finitely many of the $A_{i}$.

Dually, one gets homomorphisms from an abelian group or $R$-module $B$ to a direct product $\prod_{j \in J} C_{j}$ simply by choosing a homomorphism into each $C_{j}$; but if we wish to map $B$ into the coproduct $\bigoplus_{j \in J} C_{j} \subseteq \prod_{j \in J} C_{j}$, we face the problem of choosing those homomorphisms so that the resulting map takes each element of $B$ to an element of finite support. The question of which modules $B$ have the property that the only way to achieve this is by mapping into a finite subsum of $\bigoplus_{j \in J} C_{j}$ is answered by El Bashir, Kepka and Němec in Proposition 4.1 of [El Bashir et al. 2003]; that paper also studies the corresponding questions for colimit constructions other than coproducts.

Several workers, beginning with Chase [1962a; 1962b], have looked at the two-headed situation of module homomorphisms $f: \prod_{i \in I} A_{i} \rightarrow \bigoplus_{j \in J} C_{j}$. Here one may ask when every such map is a sum of one homomorphism which factors through the projection of $\prod_{i \in I} A_{i}$ onto a finite subproduct, and another which factors 
through the inclusion of a finite subsum in $\bigoplus_{j \in J} C_{j}$. Just as, in studying nonabelian groups in Sections 2-4, we found it desirable to divide out by $Z(B)$ to avoid certain easy ways that maps could involve infinitely many factors, so in the results of this sort, two adjustments turn out to be useful: dividing out by submodules of "highly divisible" elements of the $C_{j}$, and multiplying the given homomorphism by some nonzero ring element $d$; which essentially means restricting it to $\prod_{i \in I} d A_{i}$. Thus, [Chase 1962b, Theorem 1.2], more or less the starting point for the development of the subject, says, if restricted to the case where the base ring is $\mathbb{Z}$ and where a certain filter of principal right ideals in the statement of that theorem consists of all the nonzero ideals of $\mathbb{Z}$, that given any homomorphism of abelian groups $f: \prod_{n \in \omega} A_{n} \rightarrow \bigoplus_{i \in I} C_{i}$, there exists an integer $d>0$ such that when $f$ is applied to $\prod_{n \in \omega} d A_{n}$, and followed by the factor map $\bigoplus_{i \in I} C_{i} \rightarrow \bigoplus_{i \in I}\left(C_{i} / \bigcap_{e>0} e C_{i}\right)$, it carries the product of some cofinite subfamily of the $d A_{n}$ into the sum of a finite subfamily of the $C_{i} / \bigcap_{e>0} e C_{i}$.

That result (in its general module-theoretic form) is strengthened in [Dugas and Zimmermann-Huisgen 1981, Theorem 2] to allow products $\prod_{i \in I} A_{i}$ over any index set $I$ of cardinality less than all uncountable measurable cardinals, and to remove the requirement that the right ideals considered be principal, while in [Bergman 2006, Theorem 9], the direct product is replaced by a general inverse limit. For further related work, see references in the first paragraph of p. 46 of that paper.

(It is curious that the proof of the abovementioned theorem from [Chase 1962b], and that of Proposition 31 of this note, use virtually the same construction, but for very different purposes: in Chase's paper, to obtain a contradiction by constructing an element whose image in the direct sum would have infinitely many nonzero components; in Proposition 31, to get continuum many distinct elements in the image of our map.)

Turning back to the results of the three preceding sections, it would, of course, be desirable to investigate the corresponding questions with abelian groups replaced by modules over a general ring $R$. In [Jensen and Lenzing 1989, Chapters 7-8], algebraically compact modules over general $R$ are studied, but cotorsion modules are not mentioned. (There are numerous MathSciNet results for "cotorsion module", but I have not had time to examine them.) One might also take a hint from [Chase $1962 \mathrm{a} ; 1962 \mathrm{~b}]$, and see whether one gets nonobvious variants of our results if one considers those $B$ such that all homomorphisms $\prod_{i \in I} A_{i} \rightarrow B$ acquire the factorization properties we are looking for after multiplying $\prod_{i \in I} A_{i}$ by some integer (or ideal), and/or dividing $B$ by an appropriate subgroup (or submodule) of highly divisible elements. (This is related to the suggestion in the last paragraph of the preceding section.) 


\section{Rings}

As mentioned in Section 1, the results of this note were inspired by investigations of factorization properties of homomorphisms on direct products of notnecessarily-associative algebras over an infinite field [Bergman and Nahlus 2011; 2012; Bergman 2014; Maalouf 2014]. In those papers, the assumption of infinite base field was used to show that, under appropriate bounds on the vector-space dimension of $B$, the ultrafilters occurring had to be principal.

If we look at not-necessarily-associative rings, without assuming a structure of algebra over a field, then as we shall see below, we can still get results analogous to the main results of Section 2 (on when maps must factor through finitely many ultraproducts) and those of Section 4 (saying that such ultraproducts must be principal under appropriate assumptions on the additive structure of $B$ ). Between these we shall insert Proposition 39, which will say that if our rings have unit, then the absence of factorization through finitely many ultraproducts implies the existence of an associative commutative subring of $B$ with the cardinality of the continuum, of an explicitly describable form, over which $B$ becomes an algebra. I will not repeat here the results on algebras over an infinite field from the papers cited above; and having spent many words on those papers, I will be brief in this section.

In a direct product ring $\prod_{i \in I} R_{i}$, we define the support of an element $x=\left(x_{i}\right)_{i \in I}$ to be $\left\{i \in I \mid x_{i} \neq 0\right\}$. Whereas in Sections 2-4, our basic tool was the commutativity of elements with disjoint supports in a product group, and the phenomenon that this tool could not handle was avoided by dividing out by the center, $Z(B)$, the corresponding tool in the four works cited above was the fact that ring elements with disjoint supports have product zero; and the ideal one had to divide out by (which was also denoted $Z(B)$ ) was the zero-multiplication ideal. In this section, for $B$ a ring, we will, as in those papers, write

$$
Z(B)=\{b \in B \mid b B=B b=0\} .
$$

As in Sections 2-4, we let $\pi: B \rightarrow B / Z(B)$ be the quotient homomorphism.

There was one commutativity result in Theorem 2 above that arose for a reason other than that elements in different factors of a direct product commute, namely, (7), which followed from the fact that every element commutes with itself. Thus, the analog of that one statement, (41) below, again concerns commutativity, rather than zero products.

The obvious analog of Lemma 1 holds for rings, and yields the following analog of Theorem 2.

Theorem 37. Let $B$ be a ring (understood here to mean an abelian group given with an arbitrary bilinear multiplication $B \times B \rightarrow B$ ), and suppose there exist a family $\left(R_{i}\right)_{i \in I}$ of rings, and a surjective ring homomorphism $f: \prod_{i \in I} R_{i} \rightarrow B$, 
such that the induced homomorphism $\pi f: \prod_{i \in I} R_{i} \rightarrow B / Z(B)$ does not factor through the natural map from $\prod_{i \in I} R_{i}$ to any finite product of ultraproducts of the $R_{i}$. Then $B$ contains families of elements $\left(a_{S}\right),\left(b_{S}\right)$, indexed by the subsets $S \subseteq \omega$, such that:

(41) All the elements $a_{S}(S \subseteq \omega)$ commute with one another, and all the elements $b_{S}$ likewise commute with one another.

(42) For disjoint subsets $S$ and $T$ of $\omega$, we have $a_{S}+a_{T}=a_{S \cup T}, b_{S}+b_{T}=b_{S \cup T}$, and $0=a_{S} a_{T}=a_{S} b_{T}=b_{S} a_{T}=b_{S} b_{T}$.

(43) For subsets $S$ and $T$ of $\omega$ with $\operatorname{card}(S \cap T)=1$, we have $a_{S} b_{T} \neq 0$.

One gets from this the obvious analog of Corollary 3, which I will not write down, only noting one minor way in which the statement is weaker than that corollary: in a nonassociative ring, a family of pairwise commuting elements need not generate a commutative subring, so the assertion of commutativity in the last sentence of Corollary 3 disappears here.

One likewise has the analog of Theorem 5. Namely, following [Bergman and Nahlus 2011, Definitions 13 and 15], we define an almost direct decomposition of a ring $B$ as an expression $B=B^{\prime}+B^{\prime \prime}$, where $B^{\prime}$ and $B^{\prime \prime}$ are ideals of $B$, each of which is the 2 -sided annihilator of the other; and we shall say that $B$ has chain condition on almost direct factors if every chain of such ideals is finite. Then we get:

Theorem 38 (cf. [Bergman and Nahlus 2011, Proposition 16]). Let B again be a ring such that there exist a family of rings $\left(R_{i}\right)_{i \in I}$, and a surjective ring homomorphism $f: \prod_{i \in I} R_{i} \rightarrow B$ such that the induced homomorphism $\pi f: \prod_{i \in I} R_{i} \rightarrow$ $B / Z(B)$ does not factor through the natural map of $\prod_{i \in I} R_{i}$ to any finite direct product of ultraproducts of the $R_{i}$.

Then $B$ does not have chain condition on almost direct factors. In fact, it has a family of almost direct factors order-isomorphic to the lattice $2^{\omega}$, and forming a sublattice of the lattice of ideals of $B$.

So far we have not assumed our rings unital, since that hypothesis is unnatural for many important classes of nonassociative rings. The next result shows how in the unital case, the above theorems can be simplified and strengthened. For unital rings $B$ we have $Z(B)=0$, so $B / Z(B)$ everywhere becomes $B$. Moreover, we can take each of the systems of elements $x_{n}, y_{n} \in \prod_{i \in J_{n}} R_{i}$ from which we obtain the elements $a_{S}$ and $b_{S}$ in Theorem 37 to consist of the multiplicative identity elements of the rings $\prod_{i \in J_{n}} R_{i}$. With a little further work, we shall get:

Proposition 39. Under the common hypotheses of Theorem 37 and 38, if the rings $B$ and $R_{i}$ are unital, with homomorphisms preserving multiplicative identity elements, then $B$ is a faithful unital algebra over a commutative associative unital subring of the form $\prod_{n \in \omega} \mathbb{Z} / d_{n} \mathbb{Z}$, where each $d_{n}$ is a nonnegative integer $\neq 1$. Moreover, 
one can take all but one of the $d_{n}$ to be equal, and that one to be a multiple (not necessarily proper) of the common value of the others.

Sketch of proof. As in the proof of Theorem 2, the non-factorization of $f$ tells us that we can partition $I$ into subsets $J_{n}(n \in \omega)$ such that for each $n, f\left(\prod_{i \in J_{n}} R_{i}\right) \neq\{0\}$. (Here we regard the direct product of each subfamily of the $R_{i}$ as an ideal of $\prod_{i \in I} R_{i}$. Each of these ideals has a multiplicative identity element, generally different from that of $\prod_{i \in I} R_{i}$.) For each $S \subseteq \omega$, let $x_{S}$ denote the multiplicative identity element of $\prod_{i \in \bigcup_{n \in S} J_{n}} R_{i} \subseteq \prod_{i \in I} R_{i}$, and let $a_{S}=f\left(x_{S}\right)$. We see that the operations of multiplication by $x_{S}$ and $x_{\omega-S}$ are idempotent endomorphisms of the additive group of $\prod_{i \in I} R_{i}$, which give the projection homomorphisms to the two factors of its decomposition as

$$
\left(\prod_{i \in \cup_{n \in S} J_{n}} R_{i}\right) \times\left(\prod_{i \in \bigcup_{n \in \omega-S} J_{n}} R_{i}\right) .
$$

Hence their images $a_{S}$ and $a_{\omega-S}$ likewise determine a direct product decomposition of the ring $B$.

Now for every $S \subseteq \omega$, let $c_{S}$ denote the nonnegative integer such that the additive subgroup of $B$ generated by $a_{S}$ is isomorphic to $\mathbb{Z} / c_{S} \mathbb{Z}$ (the characteristic of the ring $\left.f\left(\prod_{i \in \bigcup_{n \in S} J_{n}} R_{i}\right)\right)$. Note that for $\varnothing \neq T \subseteq S$, we have $1 \neq c_{T} \mid c_{S}$.

The behavior of $c_{S}$ as a function of $S$ can be complicated; but with the help of the Noetherian property of the integers, we can find a family of subsets of $\omega$ on which that function has an easy description. Namely, let us choose, among all infinite $S \subseteq \omega$, one which gives a maximal value for the ideal $c_{S} \mathbb{Z}$. Then for every infinite subset $T \subseteq S$, we necessarily have $c_{T}=c_{S}$. Hence, let us partition $S$ into countably many infinite subsets, $T_{0}, \ldots, T_{m}, \ldots$, and use these to partition $\omega$ into subsets $S_{m}$, where for $m>0$, we let $S_{m}=T_{m}$, while we let $S_{0}=(\omega-S) \cup T_{0}$. Thus, for $m>0$ we have $c_{S_{m}}=c_{S}$ by choice of $S$, while $c_{S_{0}}=\operatorname{lcm}\left(c_{\omega-S}, c_{T_{0}}\right)$, a multiple of $c_{T_{0}}=c_{S}$.

Let us now map the ring $\mathbb{Z}^{\omega}$ into $\prod_{i \in I} R_{i}$ by sending each element $\left(e_{m}\right)_{m \in \omega}$ to the element whose value on each factor $\prod_{n \in S_{m}}\left(\prod_{i \in J_{n}} R_{i}\right)$ is $e_{m}$ times the multiplicative identity element, and then apply the map $f: \prod_{i \in I} R_{i} \rightarrow B$. I claim that the image of $\mathbb{Z}^{\omega}$ in $B$ will be isomorphic to $\prod_{m \in \omega} \mathbb{Z} / d_{m} \mathbb{Z}$, where $d_{m}=c_{S_{m}}$. Indeed, it is easy to verify that an element of $\mathbb{Z}^{\omega}$ that goes to zero under the componentwise map into $\prod_{m \in \omega} \mathbb{Z} / d_{m} \mathbb{Z}$ goes to zero in $B$, as a result of our choice of $S$ and the $S_{m}$. Conversely, if an element $\left(e_{m}\right)_{m \in \omega} \in \mathbb{Z}^{\omega}$ does not have zero image in $\prod_{m \in \omega} \mathbb{Z} / d_{m} \mathbb{Z}$, we can choose an $m_{0}$ such that $e_{m_{0}}$ is not divisible by $d_{m_{0}}$; and taking the image, in $B$, of the ring relation $\left(e_{m}\right)_{m \in \omega} \delta_{m_{0}}=e_{m_{0}} \delta_{m_{0}}$ in $\mathbb{Z}^{\omega}$, we see that the image of $\left(e_{m}\right)_{m \in \omega}$ in $B$ is also nonzero. 
Finally, the fact that every ring $R$ is a $\mathbb{Z}$-algebra, and that if $R$ has a multiplicative identity element $1_{R}$, its $\mathbb{Z}$-algebra structure is induced by the operations of multiplication in $R$ by members of $\mathbb{Z} \cdot 1_{R}$, easily leads to the result that $\prod_{n \in S_{m}}\left(\prod_{i \in J_{n}} R_{i}\right)$ is a $\mathbb{Z}^{\omega}$-algebra, and that this structure leads to a structure of $\prod_{m \in \omega} \mathbb{Z} / d_{m} \mathbb{Z}$-algebra on its homomorphic image $B$.

Going back to not-necessarily-unital rings, and turning to the question of when finitely many ultraproducts through which a map factors must all be principal, we can combine Theorem 25 with the idea of Lemma 12 to get the following result.

Theorem 40. Suppose $B$ is a ring which admits a surjective homomorphism from a direct product ring, $f: \prod_{i \in I} R_{i} \rightarrow B$, such that the composite

$$
\pi f: \prod_{i \in I} R_{i} \rightarrow B \rightarrow B / Z(B)
$$

factors through the product of finitely many ultraproducts of the $R_{i}$, but not through the product of finitely many countably complete ultraproducts. (So if $\operatorname{card}(I)$ is less than all uncountable measure cardinals, if any exist, the latter condition simply says that $\pi f$ does not factor through any finite product of the $A_{i}$.)

Then the additive group of $B / Z(B)$ has a nonzero cotorsion subgroup; equivalently, it either contains nonzero elements of finite order, or a copy of the additive group of $\mathbb{Q}$, or a copy of the additive group of the $p$-adic integers for some prime $p$.

\section{Monoids}

In studying homomorphisms from direct product monoids onto a monoid $B$, it is useful to assume some cancellation condition on $B$. One that will suffice for our present purposes is

$$
x y=x \Rightarrow y=e \quad \text { for } x, y \in B
$$

Note that (44) implies that one-sided inverses are two-sided, since if $x y=e$, we get $x y x=x$, which by (44) gives $y x=e$.

We shall consider two sorts of obstruction to mapping infinite products onto $B$ in ways that indiscriminately merge the factors. On the one hand, there is the same effect of noncommutativity that we took advantage of in the case of groups. On the other hand, noninvertible elements create restrictions. For instance, though linear algebra shows that the additive group $\mathbb{Q}$ admits homomorphisms from the additive group $\mathbb{Q}^{\omega}$ that behave arbitrarily on $\bigoplus_{\omega} \mathbb{Q}$, it is not hard to show that, writing $\mathbb{Q} \geq 0$ for the additive monoid of nonnegative rational numbers, it is impossible to have a homomorphism $\left(\mathbb{Q}^{\geq 0}\right)^{\omega} \rightarrow \mathbb{Q}^{\geq 0}$ that acts in a nonzero way on infinitely many of the summands of $\bigoplus_{\omega} \mathbb{Q}^{\geq 0}$. 
In our factorization results for groups, we divided out by the center of $B$; in the case of monoids, we will divide out by the group of central invertible elements. There are two versions of this concept: $Z(U(B))$, the center of the group of units (invertible elements) of $B$, and $U(Z(B))$, the group of units of the center; the former may be larger than the latter. It is $U(Z(B))$, the smaller of the two, that we will divide out by (though the other will make a brief appearance in a proof). Note that since $U(Z(B))$ consists of $B$-centralizing invertible elements, one can speak (without distinguishing right from left) of the orbits of $B$ under multiplication by that group, and the set of such orbits forms a factor monoid $B / U(Z(B))$. Clearly, the noninvertible elements of this factor monoid are precisely the cosets of the noninvertible elements of $B$. We shall write $\pi$ for the projection map $B \rightarrow B / U(Z(B))$.

Given a monoid homomorphism $f: \prod_{i \in I} M_{i} \rightarrow B$, we define the analog of the filter $\mathscr{F}$ of (2), namely

$$
\begin{aligned}
\mathscr{F}_{F}= & \left\{S \subseteq I \mid \text { the composite map } \prod_{i \in I} M_{i} \rightarrow B \rightarrow B / U(Z(B))\right. \\
& \text { factors through the projection } \left.\prod_{i \in I} M_{i} \rightarrow \prod_{i \in S} M_{i}\right\} \\
= & \left\{S \subseteq I \mid f\left(\prod_{i \in I-S} M_{i}\right) \subseteq U(Z(B))\right\} .
\end{aligned}
$$

The version of Lemma 1 that we will use for monoids is not, as for rings, a carbon copy of that lemma, so we shall give the statement and proof. (But we will cut corners where the method of proof is the same; so the reader might want to review the proof of Lemma 1 before beginning this one.) We do not yet assume the cancellativity condition (44).

Lemma 41. Let $f: \prod_{i \in I} M_{i} \rightarrow B$ be a homomorphism from a direct product of monoids $M_{i}$ to a monoid $B$, which is surjective (or more generally, such that the homomorphism $\pi f: \prod_{i \in I} M_{i} \rightarrow B \rightarrow B / U(Z(B))$ is surjective). Then the following two conditions are equivalent.

(46) The homomorphism $\pi f: \prod_{i \in I} M_{i} \rightarrow B \rightarrow B / U(Z(B))$ does not factor through the natural map $\left(\prod_{i \in I} M_{i}\right) / \mathcal{u}_{0} \times \cdots \times\left(\prod_{i \in I} M_{i}\right) / u_{n-1}$ for any finite family of ultrafilters $\cup_{0}, \ldots, \cup_{n-1}$ on $I$.

(47) There exists a partition of I into countably many subsets $J_{0}, J_{1}, \ldots$, such that either

(47a) each submonoid $\prod_{i \in J_{n}} M_{i} \subseteq \prod_{i \in I} M_{i}$ contains a pair of invertible elements $x_{n}, y_{n}$ whose images under $f$ do not commute in $B$,

or

(47b) each submonoid $\prod_{i \in J_{n}} M_{i} \subseteq \prod_{i \in I} M_{i}$ contains an element $z_{n}$ whose image in $B$ is noninvertible. 
Proof. To get (47) $\Longrightarrow$ (46), note that in the situation of (47a), since for each $n, f\left(x_{n}\right)$ and $f\left(y_{n}\right)$ are invertible and do not commute in $B$, they do not lie in $Z(U(B))$. Hence in particular $f\left(x_{n}\right) \notin U(Z(B))$, so by the final line of (45), $I-J_{n} \notin \mathscr{F}$. That this implies (46) is seen as in Lemma 1.

If, rather, we are in the situation of (47b), then the fact that the $f\left(z_{n}\right)$ are nonunits implies that they do not lie in $U(Z(B))$, giving the same result for the same reason.

The proof of the converse begins, as for Lemma 1, with the observation that (46) implies that there exists a partition of $I$ into countably many subsets $J_{0}, J_{1}, \ldots$, such that each $\prod_{i \in J_{n}} M_{i}$ contains elements mapped by $f$ to elements of $B$ not in $U(Z(B))$. Let $L_{n}=\prod_{i \in J_{n}} M_{i}$, so that $\prod_{i \in I} M_{i}=\prod_{n \in \omega} L_{n}$. Clearly, it will either be true that for infinitely many $n$, the submonoid $f\left(L_{n}\right) \subseteq B$ contains a noninvertible element, or that for infinitely many $n$, that submonoid consists entirely of invertible elements.

In the former case, those $J_{n}$ such that $f\left(L_{n}\right)$ contains a noninvertible element of $B$ will constitute a partition of some subset $J \subseteq I$ into countably many subsets. If we enlarge one of these sets by throwing in the complementary set $I-J$, we get a partition of $I$ of the sort described in (47b).

If, on the other hand, there are infinitely many $n$ such that $f\left(L_{n}\right)$ consists entirely of invertible elements of $B$, then for each such $n$, let us choose an $x_{n} \in L_{n}$ such that $f\left(x_{n}\right) \notin U(Z(B))$, and then a $y \in \prod_{m \in \omega} L_{m}$ such that $f(y)$ does not commute with $f\left(x_{n}\right)$. As in the proof of Lemma 1, we can obtain from $y$ an element $y_{n} \in L_{n}$ such that $f\left(y_{n}\right)$ still does not commute with $f\left(x_{n}\right)$. By assumption, $f\left(L_{n}\right)$ consists of invertible elements, so $f\left(x_{n}\right)$ and $f\left(y_{n}\right)$ belong to $U(B)$. Thus, we have a partition of some $J \subseteq I$ into countably many subsets as in (47a). Again tacking $I-J$ onto one of these, we can take this to be a partition of the whole set $I$.

This leads to an analog of Theorem 2 which, similarly, has two alternative conclusions. We shall describe one of these by referring to that earlier theorem, and spell out the other.

Theorem 42. Let $B$ be a monoid satisfying the cancellation condition (44), and suppose there exists a family $\left(M_{i}\right)_{i \in I}$ of monoids, and a monoid homomorphism $f$ : $\prod_{i \in I} M_{i} \rightarrow B$ such that the induced homomorphism $\pi f: \prod_{i \in I} M_{i} \rightarrow B / U(Z(B))$ does not factor through any finite product of ultraproducts of the $M_{i}$.

Then either

(a) the group $U(B)$ satisfies the hypothesis, and hence the conclusions, of Theorem 2,

or

(b) B contains a family of elements $\left(a_{S}\right)$ indexed by the subsets $S \subseteq \omega$ and satisfying the following conditions:

(48) $a_{\varnothing}=e$, and all the elements $a_{S}(S \subseteq \omega)$ commute with one another. 
(49) For disjoint sets $S, T \subseteq \omega$, one has $a_{S} a_{T}=a_{S \cup T}$.

(50) For sets $S \subsetneq T \subseteq \omega, a_{T}$ is a right multiple of $a_{S}$, but $a_{S}$ is not a right multiple of $a_{T}$.

Proof. The two cases of (47) will yield the two alternative conclusions shown. It is easy to verify that (47a) yields conclusion (a).

In case (47b), let $L_{n}=\prod_{i \in J_{n}} M_{i}$, and take elements $z_{n} \in L_{n}$ with noninvertible images in $B$. For each $S \subseteq \omega$, let $a_{S}$ be the image under $f$ of the element of $\prod_{n \in S} L_{n} \subseteq \prod_{n \in \omega} L_{n}$ whose $n$-th coordinate is $z_{n}$ for each $n \in S$. Then (48) and (49) are immediate, and the first assertion of (50) follows from (49) applied to $S$ and $T-S$.

To get the final assertion of (50), choose any $n \in T-S$, and note that by (49), we have

$$
a_{T}=a_{S} a_{T-(S \cup\{n\})} a_{\{n\}} .
$$

If we also had $a_{S}=a_{T} b$ for some $b \in B$, then substituting this into the righthand-side of (51) and canceling $a_{T}$ by (44), we could conclude that $a_{\{n\}}$ was left invertible, hence by the observation following (44), invertible, contradicting our choice of $z_{n}$.

In case (b) of Theorem 42, we cannot say, as we can in case (a), that distinct sets $S$ yield distinct elements $a_{S} \in B$. For instance, let $B$ be the factor monoid of the additive monoid $\left(\mathbb{Z}^{\geq 0}\right)^{\omega}$ by the relation that equates elements $x$ and $y$ if there is some $n \geq 0$ such that $x$ and $y$ agree at all but the first $n$ coordinates, and such that the sum of the entries at those first $n$ coordinates is the same for $x$ and $y$. Then $B$ is a cancellative abelian monoid with trivial group of units, and the quotient map $f:\left(\mathbb{Z}^{\geq 0}\right)^{\omega} \rightarrow B$ does not annihilate any of the $\delta_{n}$ (defined in $\left(\mathbb{Z}^{\geq 0}\right)^{\omega}$ as in $\mathbb{Z}^{\omega}$ ). Hence (47b) holds for this $f$, with the $J_{n}$ taken to be the singletons $\{n\}$, and $z_{n}=\delta_{n}$. But defining the $a_{S}$ in terms of these as in the proof of Theorem 42, we find that for finite subsets $S, T \subseteq \omega$ of the same cardinality, we have $a_{S}=a_{T}$ in $B$; so the $a_{S}$ are not all distinct.

Nevertheless, in the situation of Theorem 42(b) we always get continuum many distinct $a_{S}$. For by (50), distinct comparable sets give distinct elements; and the partially ordered set of subsets of any countably infinite set has chains of the order type of the real numbers. (Indeed, the countable set of rational numbers has the chain of Dedekind cuts, and any countably infinite set can be put in bijective correspondence with the rationals.) Thus, we get:

Corollary 43. In the situation of conclusion (b) of Theorem 42, B has a set of mutually commuting noninvertible elements which form, under the relation of divisibility, a chain with the order type of the real numbers. In particular, $B$ (and in fact, $B / U(Z(B))$ has at least the cardinality of the continuum. 
The results proved above are far from optimal. For instance, the conclusions of Theorem 42 and Corollary 43 are consistent with $B$ being the additive monoid $\mathbb{R}^{\geq 0}$ of nonnegative real numbers; but that case is easy to exclude. Indeed, suppose $B=\mathbb{R}^{\geq 0}$ admitted a map as in the hypothesis of Theorem 42. By the proof of Lemma 41 , since $B$ has trivial group of units, we must have a homomorphism $f: \prod_{n \in \omega} L_{n} \rightarrow B$ such that each $L_{n}$ has an element $x_{n}$ making $f\left(x_{n}\right)$ a positive real number. By the Archimedean property of the reals, we can modify our choices of $x_{n}$ so that for each $n$ we have $f\left(x_{n}\right) \geq 1$. Thus, when we construct elements $a_{S} \in B$ as in the proof of the theorem, we get $a_{\{n\}} \geq 1$ for each $n$, from which it follows that for any $S \subseteq \omega$ of $\geq m$ elements $(m \in \omega), a_{S} \geq m$. For $S$ infinite, this gives a contradiction; so $B$ admits no such map. It is not clear to me what the best assertion that can be gotten by this technique is.

Let us also note that in place of the two-way subdivision of the sets $J_{n}$ used in the proof of Lemma 41, we could (at least if we assumed full cancellativity rather than just (44)) have used a three-way subdivision, noting that for each $n, f\left(L_{n}\right)$ either contains noncommuting invertible elements of $B$, or contains noncommuting noninvertible elements, or contains a central noninvertible element. (Cancellativity is needed to show that if a nonunit $x$ and a unit $u$ fail to commute, then so do the two nonunits $x$ and $x u$.) So there must be infinitely many $n$ for which one of these statements holds, and we can deduce a three-alternative conclusion: either, as before, we have invertible elements $a_{S}, b_{S} \in B$ indexed by the subsets $S \subseteq \omega$ which can be distinguished by their commutativity relations, or we have elements $a_{S}, b_{S} \in B$ which, except for $a_{\varnothing}, b_{\varnothing}$, are noninvertible, and satisfy the same relations and can be distinguished in the same way, or we have central elements $a_{S}$ which satisfy (48)-(50).

Though one could define "almost direct factors" for monoids, as for groups, using submonoids that are each other's centralizers, there doesn't seem to be an analogous way to "split" a monoid based on noninvertible central elements; so I have not attempted to formulate an analog of Theorem 5. I leave further exploration of these questions to those better versed than I in the study of monoids.

One can also consider for semigroups the same factorization properties studied here for monoids. Since the above constructions involved elements of direct products defined to have the value $e$ on complements of given subsets $S$ of our index set, the absence of identity elements should lead to changes in what can be proved.

\section{Lattices: a case worth looking at}

One other class of mathematical structures suggests itself, to which similar methods might be applicable - lattices. Just as a direct product decomposition of a group or monoid leads to certain pairs of elements that must commute, and a direct product 
decomposition of a ring leads to certain pairs of elements that must have zero product, so a direct product decomposition of a lattice leads to certain 3-tuples of elements that must satisfy distributivity relations. Perhaps this observation can be used to get lattice-theoretic analogs of some of the results of this note.

(In [Bergman 2014, §5] I speculate on very general properties of a variety of algebras that would allow one to get such results; but I am not confident that that approach will go anywhere.)

\section{Note added in proof}

Jan Šaroch (personal communication) has obtained a positive answer to Question 33, which applies more generally to a module $B$ over any countable ring $R$ such that $R^{\omega}$ is a flat Mittag-Leffler module.

\section{Acknowledgements}

I am indebted to Thomas Scanlon for suggesting Lemma 11, to M. Dugas, L. Fuchs, K. M. Rangaswamy and J. Rotman for helpful correspondence regarding Sections 5 and 6 , and to the referee for several useful suggestions.

\section{References}

[Bergman 1972] G. M. Bergman, "Boolean rings of projection maps", J. London Math. Soc. (2) 4 (1972), 593-598. MR 47 \#93 Zbl 0205.03805

[Bergman 2006] G. M. Bergman, "Two statements about infinite products that are not quite true", pp. 35-58 in Groups, rings and algebras, edited by W. Chin et al., Contemp. Math. 420, Amer. Math. Soc., Providence, RI, 2006. MR 2007k:16008 Zbl 1129.16002

[Bergman 2014] G. M. Bergman, "Families of ultrafilters, and homomorphisms on infinite direct product algebras”, J. Symb. Log. 79:1 (2014), 223-239. MR 3226022 Zbl 06349658

[Bergman and Nahlus 2011] G. M. Bergman and N. Nahlus, "Homomorphisms on infinite direct product algebras, especially Lie algebras", J. Algebra 333 (2011), 67-104. MR 2012f:17005 Zbl 1276.17002

[Bergman and Nahlus 2012] G. M. Bergman and N. Nahlus, "Linear maps on $k^{I}$, and homomorphic images of infinite direct product algebras", J. Algebra 356 (2012), 257-274. MR 2891132 Zbl 1260.15003

[Chang and Keisler 1990] C. C. Chang and H. J. Keisler, Model theory, 3rd ed., Studies in Logic and the Foundations of Mathematics 73, North-Holland, Amsterdam, 1990. MR 91c:03026 Zbl 0697.03022

[Chase 1962a] S. U. Chase, "A remark on direct products of modules", Proc. Amer. Math. Soc. 13 (1962), 214-216. MR 25 \#1197 Zbl 0111.04101

[Chase 1962b] S. U. Chase, "On direct sums and products of modules", Pacific J. Math. 12 (1962), 847-854. MR 26 \#2472 Zbl 0115.26002

[Comfort and Negrepontis 1974] W. W. Comfort and S. Negrepontis, The theory of ultrafilters, Die Grundlehren der mathematischen Wissenschaften 211, Springer, New York, 1974. MR 53 \#135 Zbl 0298.02004 
[Dugas and Göbel 1982] M. Dugas and R. Göbel, "Every cotorsion-free ring is an endomorphism ring”, Proc. London Math. Soc. (3) 45:2 (1982), 319-336. MR 84b:20064 Zbl 0506.16022

[Dugas and Zimmermann-Huisgen 1981] M. Dugas and B. Zimmermann-Huisgen, "Iterated direct sums and products of modules", pp. 179-193 in Abelian group theory (Oberwolfach, 1981), edited by R. Göbel and E. Walker, Lecture Notes in Math. 874, Springer, Berlin, 1981. MR 83f:16059 Zbl 0475.13011

[Eklof 1973] P. C. Eklof, "The structure of ultraproducts of abelian groups”, Pacific J. Math. 47 (1973), 67-79. MR 48 \#6281 Zbl 0244.20063

[El Bashir et al. 2003] R. El Bashir, T. Kepka, and P. Němec, "Modules commuting (via Hom) with some colimits”, Czechoslovak Math. J. 53:4 (2003), 891-905. MR 2004i:16002 Zbl 1080.16504

[Fuchs 1970] L. Fuchs, Infinite abelian groups, vol. I, Pure and Applied Mathematics 36, Academic Press, New York, 1970. MR 41 \#333 Zbl 0209.05503

[Fuchs 1973] L. Fuchs, Infinite abelian groups, vol. II, Pure and Applied Mathematics 36, Academic Press, New York, 1973. MR 50 \#2362 Zbl 0257.20035

[Jensen and Lenzing 1989] C. U. Jensen and H. Lenzing, Model-theoretic algebra with particular emphasis on fields, rings, modules, Algebra, Logic and Applications 2, Gordon and Breach, New York, 1989. MR 91m:03038 Zbl 0728.03026

[Lam 1999] T. Y. Lam, Lectures on modules and rings, Graduate Texts in Mathematics 189, Springer, New York, 1999. MR 99i:16001 Zbl 0911.16001

[Maalouf 2014] F. Maalouf, "Weakly slender algebras", J. Pure Appl. Algebra 218:9 (2014), 17541759. MR 3188870 Zbl 06280189

[Muranov 2007] A. Muranov, "Finitely generated infinite simple groups of infinite commutator width”, Internat. J. Algebra Comput. 17:3 (2007), 607-659. MR 2009f:20040 Zbl 1141.20022

[Nunke 1961] R. J. Nunke, "Slender groups", Bull. Amer. Math. Soc. 67 (1961), 274-275. MR 24 \#A163 Zbl 0099.01301

[Rotman 2009] J. J. Rotman, An introduction to homological algebra, 2nd ed., Springer, New York, 2009. MR 2009i:18011 Zbl 1157.18001

[Shelah and Strüngmann 2001] S. Shelah and L. Strüngmann, "The failure of the uncountable noncommutative Specker phenomenon", J. Group Theory 4:4 (2001), 417-426. MR 2002g:20049 Zbl 0998.20026

Received June 7, 2014. Revised October 2, 2014.

GEORGE M. BERGMAN

DEPARTMENT OF MATHEMATICS

UNIVERSITY OF CALIFORNIA

BERKELEY, CA 94720-3840

UNITED STATES

gbergman@math.berkeley.edu 


\title{
PACIFIC JOURNAL OF MATHEMATICS
}

\author{
msp.org/pjm
}

Founded in 1951 by E. F. Beckenbach (1906-1982) and F. Wolf (1904-1989)

\section{EDITORS}

Don Blasius (Managing Editor)

Department of Mathematics

University of California

Los Angeles, CA 90095-1555

blasius@math.ucla.edu

\author{
Paul Balmer \\ Department of Mathematics \\ University of California \\ Los Angeles, CA 90095-1555 \\ balmer@math.ucla.edu \\ Robert Finn \\ Department of Mathematics \\ Stanford University \\ Stanford, CA 94305-2125 \\ finn@math.stanford.edu \\ Sorin Popa \\ Department of Mathematics \\ University of California \\ Los Angeles, CA 90095-1555 \\ popa@math.ucla.edu
}

\author{
Vyjayanthi Chari \\ Department of Mathematics \\ University of California \\ Riverside, CA 92521-0135 \\ chari@math.ucr.edu \\ Kefeng Liu \\ Department of Mathematics \\ University of California \\ Los Angeles, CA 90095-1555 \\ liu@math.ucla.edu \\ Jie Qing \\ Department of Mathematics \\ University of California \\ Santa Cruz, CA 95064 \\ qing@ cats.ucsc.edu
}

\section{PRODUCTION}

Silvio Levy, Scientific Editor, production@msp.org

\section{SUPPORTING INSTITUTIONS}

ACADEMIA SINICA, TAIPEI

CALIFORNIA INST. OF TECHNOLOGY

INST. DE MATEMÁTICA PURA E APLICADA

KEIO UNIVERSITY

MATH. SCIENCES RESEARCH INSTITUTE

NEW MEXICO STATE UNIV.

OREGON STATE UNIV.

\author{
STANFORD UNIVERSITY \\ UNIV. OF BRITISH COLUMBIA \\ UNIV. OF CALIFORNIA, BERKELEY \\ UNIV. OF CALIFORNIA, DAVIS \\ UNIV. OF CALIFORNIA, LOS ANGELES \\ UNIV. OF CALIFORNIA, RIVERSIDE \\ UNIV. OF CALIFORNIA, SAN DIEGO \\ UNIV. OF CALIF., SANTA BARBARA
}

\author{
Daryl Cooper \\ Department of Mathematics \\ University of California \\ Santa Barbara, CA 93106-3080 \\ cooper@math.ucsb.edu \\ Jiang-Hua Lu \\ Department of Mathematics \\ The University of Hong Kong \\ Pokfulam Rd., Hong Kong \\ jhlu@maths.hku.hk \\ Paul Yang \\ Department of Mathematics \\ Princeton University \\ Princeton NJ 08544-1000 \\ yang@math.princeton.edu
}

These supporting institutions contribute to the cost of publication of this Journal, but they are not owners or publishers and have no responsibility for its contents or policies.

See inside back cover or msp.org/pjm for submission instructions.

The subscription price for 2015 is US \$420/year for the electronic version, and \$570/year for print and electronic.

Subscriptions, requests for back issues and changes of subscribers address should be sent to Pacific Journal of Mathematics, P.O. Box 4163, Berkeley, CA 94704-0163, U.S.A. The Pacific Journal of Mathematics is indexed by Mathematical Reviews, Zentralblatt MATH, PASCAL CNRS Index, Referativnyi Zhurnal, Current Mathematical Publications and Web of Knowledge (Science Citation Index).

The Pacific Journal of Mathematics (ISSN 0030-8730) at the University of California, c/o Department of Mathematics, 798 Evans Hall \#3840, Berkeley, CA 94720-3840, is published twelve times a year. Periodical rate postage paid at Berkeley, CA 94704, and additional mailing offices. POSTMASTER: send address changes to Pacific Journal of Mathematics, P.O. Box 4163, Berkeley, CA 94704-0163.

PJM peer review and production are managed by EditFLOW ${ }^{\circledR}$ from Mathematical Sciences Publishers.

\section{PUBLISHED BY}

\section{mathematical sciences publishers \\ nonprofit scientific publishing}

http://msp.org/

(C) 2015 Mathematical Sciences Publishers 


\section{PACIFIC JOURNAL OF MATHEMATICS}

Volume $274 \quad$ No. $2 \quad$ April 2015

On Demazure and local Weyl modules for affine hyperalgebras

Angelo Bianchi, Tiago Macedo and Adriano Moura

On curves and polygons with the equiangular chord property

305

TARik Aougab, Xidian Sun, Serge TabachniKov and

YUWEN WANG

The well-posedness of nonlinear Schrödinger equations in Triebel-type 325 spaces

SHAOLEI RU and JiECHENG CHEN

Hypersurfaces with constant curvature quotients in warped product manifolds

JIE WU and CHAO XIA

The first terms in the expansion of the Bergman kernel in higher degrees

MARTin PUCHOL and JiAlin ZHU

Determinant rank of $C^{*}$-algebras

Guinua Gong, HuAXIN Lin and Yifeng XUE

Motion by mixed volume preserving curvature functions near spheres

437

DAVID HARTLEY

Homomorphisms on infinite direct products of groups, rings and monoids

GEORGE M. BERGMAN

The virtual first Betti number of soluble groups

Martin R. BRIDSON and Dessislava H. KochloukOVA 